\title{
Parabolic Harnack inequality and estimates of Markov chains on graphs
}

Thierry Delmotte

Abstract. On a graph, we give a characterization of a parabolic Harnack inequality and Gaussian estimates for reversible Markov chains by geometric properties (volume regularity and Poincaré inequality).

\section{Introduction.}

Consider the standard random walk with kernel $p_{n}(x, y)$ on a graph $\Gamma$ with polynomial volume growth. Under which conditions does one have the following Gaussian estimates?

$$
\frac{c}{V(x, \sqrt{n})} e^{-C d(x, y)^{2} / n} \leq p_{n}(x, y) \leq \frac{C}{V(x, \sqrt{n})} e^{-c d(x, y)^{2} / n}
$$

where $V(x, n)$ is the cardinal of the ball of center $x$ and radius $n$. Note first that $p_{n}(x, y)$ may be null for $d(x, y)>n$ or for $d(x, y) \not \equiv n(\bmod 2)$. Thus we will consider only $d(x, y) \leq n$ and graphs where all vertices are loops. With these precisions, the Gaussian estimates were proved when $\Gamma$ is a group in [15]. They were also proved for linear volume growth in [5] and it was there conjectured that they were true for polynomial growth under an isoperimetric assumption such as Poincaré inequality. Indeed in the continuous setting of Riemannian manifolds, they were 
proved first for non-negative Ricci curvature in [19] and then under Poincaré inequality assumption in [12], [30]. All these proofs are based on a parabolic Harnack inequality and [30] shows that the Poincaré inequality is the good isoperimetric assumption.

The aim of this paper, which is announced in [10], is to prove the conjecture in [5] and more precisely to give a characterization of the parabolic Harnack inequality or the Gaussian estimates by geometric properties (volume regularity and Poincaré inequality) which is the discrete counterpart of the main result in [30]. A precise statement is proposed in Section 1.4 after some definitions. The main part, the proof of the Harnack inequality, is an application of J. Moser's method [21], [22], [23]. His approach is presented on Euclidian spaces $\mathbb{R}^{n}$ but shows clearly the contribution of Poincaré and Sobolev inequalities. That's why it has been adapted to many different settings.

As far as graphs are concerned, elliptic versions (without the time variable) of the Harnack inequality have been proved in [20] with a special isoperimetric assumption and in [9], [16], [26] by J. Moser's iterative method with Poincaré inequality. The discretization of the space raised some technical problems but the proof could go through. It is much more intricate to deal with both discretizations (space and time), especially to obtain Cacciopoli inequalities. Section 1.5 is an attempt to show these difficulties and their origin. Because of these criss-crossing discretizations difficulties, we have tried to prove a continuous-time parabolic Harnack inequality on graphs and this raised only solvable technical problems like the elliptic version.

One application of the Harnack inequality is another proof of Hölder regularity (see Section 4.1) for solutions of the elliptic/parabolic equation (theorem of J. Nash [24]). Another application is that it yields Gaussian estimates. The study of these estimates in the mixed setting (discrete geometry, continuous time) in [8], [25] has been helpful because at first we only prove the Harnack inequality in this setting.

At this Gaussian estimates step, it is possible to deduce discretetime results from the continuous-time ones. This is the crucial point of this paper because all other steps are more or less adaptations of known technics which are fully reviewed in [30]. This strategy -to work on the continuous-time setting and to compare with the discrete-time settingis also employed in [31, Section 1.4.1]. One side of the comparison is stated in Theorem 3.6 and may be used again. The other side will depend closely on the problem considered.

To deduce Gaussian estimates from the Harnack inequality is the 
classical (chronological after J. Moser's work) way to introduce this theory. The reverse order based on J. Nash's ideas [24] and completed in [11] is also useful here because our discrete-time Gaussian estimates yield the discrete-time Harnack inequality, which is finally proven after a return trip to Gaussian estimates.

Let us note that we aimed at not using any algebraic structure (and we get an equivalence, which proves that this structure plays no role in fact). Similarly, the authors of [3] tried to extend related results to a more general class than Cayley graphs (strongly convex subgraphs of homogeneous graphs) and in [33] some estimates are obtained still on the particular graphs $\mathbb{Z}^{n}$ (with a continuous time) but for non-uniform transitions.

\subsection{The geometric setting.}

Let $\Gamma$ be an infinite set and $\mu_{x y}=\mu_{y x} \geq 0$ a symmetric weight on $\Gamma \times \Gamma$. It induces a graph structure if we call $x$ and $y$ neighbours $(x \sim y)$ when $\mu_{x y} \neq 0$ (note that loops are allowed). We will assume that this graph is connected and locally uniformly finite - this means there exists $N$, such that for all $x \in \Gamma, \#\{y: y \sim x\} \leq N$ and it is implied by the geometric conditions (see below) $D V\left(C_{1}\right)$ or $\Delta(\alpha)$. Vertices are weighted by $m(x)=\sum_{y \sim x} \mu_{x y}$. The graph is endowed with its natural metric (the smallest number of edges of a path between two points). We define balls (for $r$ real) $B(x, r)=\{y: d(x, y) \leq r\}$ and the volume of a subset $A$ of $\Gamma, V(A)=\sum_{x \in A} m(x)$. We will write $V(x, r)$ for $V(B(x, r))$.

We shall consider the following geometric conditions:

Definition 1.1. The weighted graph $(\Gamma, \mu)$ satisfies the volume regularity (or doubling volume property) $D V\left(C_{1}\right)$ if

$$
V(x, 2 r) \leq C_{1} V(x, r), \quad \text { for all } x \in \Gamma, \text { for all } r \in \mathbb{R}^{+} .
$$

This implies for $r \geq s$ that (the square brackets denote the integer part)

$$
\begin{aligned}
V(x, r) & \leq V\left(x, 2^{[\log (r / s) / \log 2]+1} s\right) \\
& \leq C_{1} C_{1}^{\log (r / s) / \log 2} V(x, s) \\
& =C_{1}\left(\frac{r}{s}\right)^{\log C_{1} / \log 2} V(x, s) .
\end{aligned}
$$


Definition 1.2. The weighted graph $(\Gamma, \mu)$ satisfies the Poincaré inequality $P\left(C_{2}\right)$ if

$$
\sum_{x \in B\left(x_{0}, r\right)} m(x)\left|f(x)-f_{B}\right|^{2} \leq C_{2} r^{2} \sum_{x, y \in B\left(x_{0}, 2 r\right)} \mu_{x y}(f(y)-f(x))^{2},
$$

for all $f \in \mathbb{R}^{\Gamma}$, for all $x_{0} \in \Gamma$, for all $r \in \mathbb{R}^{+}$, where

$$
f_{B}=\frac{1}{V\left(x_{0}, r\right)} \sum_{x \in B\left(x_{0}, r\right)} m(x) f(x) .
$$

Some methods to obtain this Poincaré inequality on a graph are proposed in [6].

Definition 1.3. Let $\alpha>0,(\Gamma, \mu)$ satisfies $\Delta(\alpha)$ if

$$
x \sim y \text { implies } \mu_{x y} \geq \alpha m(x), \quad \text { for all } x \in \Gamma, x \sim x
$$

Two assertions are contained in this definition. The fact that $\mu_{x y} \neq 0$ implies $\mu_{x y} \geq \alpha m(x)$ is a local ellipticity property -it may be understood as a local volume regularity if we see the graph as a network. It implies that the graph is locally uniformly finite with $N=1 / \alpha$ (so does also $D V\left(C_{1}\right)$ with $\left.N=C_{1}^{2}\right)$. Only this first assertion is needed for continuous-time results (such as Theorem 2.1). The second assertion is that $\mu_{x x} \geq \alpha m(x)$ (or $p(x, x) \geq \alpha$ with the notations of next section). It will be used in Section 3.2 to compare the discrete-time and continuous-time Markov kernels. This condition appears in [4] where the authors prove that it implies the analyticity of the Markov operator. This fact is used for instance in [27] to obtain temporal regularity.

If one considers a weighted graph $(\Gamma, \mu)$ which satisfies only the first assertion, for instance the standard random walk on $\mathbb{Z}\left(\mu_{m n}=1\right.$ if $|m-n|=1, \mu_{m n}=0$ otherwise), one can study the graph $\left(\Gamma, \mu^{(2)}\right)$ where

$$
\mu_{x y}^{(2)}=\sum_{z} \frac{\mu_{x z} \mu_{z y}}{m(z)} .
$$

With the notations of next section, this gives the iterated kernel

$$
p^{(2)}(x, y)=p_{2}(x, y) \quad \text { and } \quad m^{(2)}(x)=m(x) .
$$


The point is that

$$
\mu_{x x}^{(2)}=\sum_{z} \frac{\left(\mu_{x z}\right)^{2}}{m(z)} \geq \sum_{z \sim x} \alpha \mu_{x z}=\alpha m(x) .
$$

Thus $\left(\Gamma, \mu^{(2)}\right)$ satisfies the complete assumption $\Delta\left(\alpha^{2}\right)$. Indeed, if $\mu_{x y}^{(2)} \neq 0$, there exists $z_{0}$ such that $\mu_{x z_{0}} \neq 0$ and $\mu_{z_{0} y} \neq 0$ and

$$
\mu_{x y}^{(2)} \geq \frac{\mu_{x z_{0}} \mu_{z_{0} y}}{m\left(z_{0}\right)} \geq \alpha \mu_{x z_{0}} \geq \alpha^{2} m(x) .
$$

To extract afterwards from the results for $\left(\Gamma, \mu^{(2)}\right)$ some consequences for $(\Gamma, \mu)$, we must be careful that $\left(\Gamma, \mu^{(2)}\right)$ may not be connected (see the standard random walk on $\mathbb{Z})$.

\subsection{Markov chains and parabolic equations.}

To the weighted graph we associate discrete-time and continuoustime reversible Markov kernels. Set $p(x, y)=\mu_{x y} / m(x)$, the discrete kernel $p_{n}(x, y)$ is defined by

$$
\left\{\begin{array}{l}
p_{0}(x, z)=\delta(x, z), \\
p_{n+1}(x, z)=\sum_{y} p(x, y) p_{n}(y, z) .
\end{array}\right.
$$

This kernel is not symmetric but

$$
\frac{p_{n}(x, y)}{m(y)}=\frac{p_{n}(y, x)}{m(x)} .
$$

We keep this notation which represents the probability to go from $x$ to $y$ in $n$ steps but it may also be interesting to think to the density

$$
h_{n}(x, y)=\frac{p_{n}(x, y)}{m(y)}
$$

which is symmetric and is the right analog of a kernel on a continuous space.

We will say that $u$ satisfies the (discrete-time) parabolic equation on $(n, x)$ if

$$
m(x) u(n+1, x)=\sum_{y} \mu_{x y} u(n, y)
$$


It is the case of $p \cdot(\cdot, y)$.

Note that we have a weighted geometry and we consider only the canonic parabolic equation on it. Imagine we had a non-weighted geometry (this means volume regularity and Poincaré inequality without the weights $m(x)$ and $\mu_{x y}$ ) and any parabolic equation with a uniform ellipticity constant, then we would consider the geometry weighted by the coefficients of the equation. Because of the ellipticity, the geometric assumptions on the non-weighted geometry would yield those on the weighted geometry. Our background is a little more general since the ellipticity constant $(\Delta(\alpha))$ is not uniform but above all, as A. Grigor'yan pointed out to us, its presentation is cleaner because these geometric assumptions are always applied with the weights.

Note also that every reversible Markov chain can be obtained as above: starting from the Markov kernel $p$ and its invariant measure $m$, one constructs $\mu_{x y}=p(x, y) m(x)$.

Definition 1.4. The weighted graph $(\Gamma, \mu)$ satisfies the Gaussian estimates $G\left(c_{l}, C_{l}, C_{r}, c_{r}\right)$ (all constants are positive) if

$$
\begin{aligned}
d(x, y) \leq n \text { implies } \frac{c_{l} m(y)}{V(x, \sqrt{n})} e^{-C_{l} d(x, y)^{2} / n} & \leq p_{n}(x, y) \\
& \leq \frac{C_{r} m(y)}{V(x, \sqrt{n})} e^{-c_{r} d(x, y)^{2} / n} .
\end{aligned}
$$

Of course, if $d(x, y)>n$ then $p_{n}(x, y)=0$. On Euclidian spaces, these estimates were first proved for fundamental solutions of parabolic equations in [1].

The continuous-time Markov kernel may be defined by

$$
\mathcal{P}_{t}(x, z)=e^{-t} \sum_{k=0}^{+\infty} \frac{t^{k}}{k !} p_{k}(x, z) .
$$

Like the discrete kernel, it satisfies

$$
\frac{\mathcal{P}_{t}(x, y)}{m(y)}=\frac{\mathcal{P}_{t}(y, x)}{m(x)} .
$$

It is also the solution for $(t, x) \in \mathbb{R}^{+} \times \Gamma$ of

$$
\left\{\begin{array}{l}
\mathcal{P}_{0}(x, z)=\delta(x, z) \\
m(x) \frac{\partial}{\partial t} \mathcal{P}_{t}(x, z)=\sum_{y} \mu_{x y}\left(\mathcal{P}_{t}(y, z)-\mathcal{P}_{t}(x, z)\right) .
\end{array}\right.
$$


Indeed,

$$
\begin{aligned}
\frac{\partial}{\partial t} \mathcal{P}_{t}(x, z) & =e^{-t}\left(\sum_{k=1}^{+\infty} \frac{k t^{k-1}}{k !} p_{k}(x, z)-\sum_{k=0}^{+\infty} \frac{t^{k}}{k !} p_{k}(x, z)\right) \\
& =e^{-t}\left(\sum_{k=1}^{+\infty} \frac{t^{k-1}}{(k-1) !} \sum_{y} p(x, y) p_{k-1}(y, z)-\sum_{k=0}^{+\infty} \frac{t^{k}}{k !} p_{k}(x, z)\right) \\
& =\sum_{y} p(x, y)\left(\mathcal{P}_{t}(y, z)-\mathcal{P}_{t}(x, z)\right) .
\end{aligned}
$$

Therefore we will say that $u$ satisfies the (continuous-time) parabolic equation on $(t, x)$ if

$$
m(x) \frac{\partial}{\partial t} u(t, x)=\sum_{y} \mu_{x y}(u(t, y)-u(t, x)) .
$$

\subsection{Parabolic Harnack inequalities.}

These inequalities apply to positive solutions of the parabolic equations on cylinders (products of a time interval and a ball). Let us make this precise on the boundary of the cylinders. We shall say that $u$ is a non-negative solution on $Q=I \times B\left(x_{0}, r\right)$ if it is the trace of a non-negative solution on $I \times B\left(x_{0}, r+1\right)$ which satisfies (1.2) or (1.3) everywhere on $Q$. For instance in the continuous case, this implies: for all $(t, x) \in Q$,

$$
u(t, x) \geq 0,
$$

for all $t \in I$, for all $x \in B\left(x_{0}, r-1\right)$,

$$
m(x)\left(\frac{\partial}{\partial t} u(t, x)+u(t, x)\right)=\sum_{y} \mu_{x y} u(t, y),
$$

for all $t \in I, d\left(x_{0}, x\right)=[r]$ imply

$$
m(x)\left(\frac{\partial}{\partial t} u(t, x)+u(t, x)\right) \geq \sum_{y \in B\left(x_{0}, r\right)} \mu_{x y} u(t, y) .
$$


Definition 1.6. Set $\eta \in] 0,1\left[\right.$ and $0<\theta_{1}<\theta_{2}<\theta_{3}<\theta_{4},(\Gamma, \mu)$ satisfies the continuous-time parabolic Harnack inequality

$$
\mathcal{H}\left(\eta, \theta_{1}, \theta_{2}, \theta_{3}, \theta_{4}, C\right)
$$

if for all $x_{0}, s, r$ and every non-negative solution on $Q=\left[s, s+\theta_{4} r^{2}\right] \times$ $B\left(x_{0}, r\right)$, we have

$$
\sup _{Q_{\ominus}} u \leq C \inf _{Q_{\oplus}} u
$$

where $Q_{\ominus}=\left[s+\theta_{1} r^{2}, s+\theta_{2} r^{2}\right] \times B\left(x_{0}, \eta r\right)$ and $Q_{\oplus}=\left[s+\theta_{3} r^{2}, s+\right.$ $\left.\theta_{4} r^{2}\right] \times B\left(x_{0}, \eta r\right)$.

Let us explain the choice of the boundary condition. For $r<1, Q$ has no interior so we just have (1.4) but this is sufficient to obtain the inequality since it gives a lower bound for $(\partial / \partial t) u\left(t, x_{0}\right)$.

If we assume $\Delta(\alpha)$ and $\mathcal{H}\left(\eta, \theta_{1}, \theta_{2}, \theta_{3}, \theta_{4}, C\right)$, then for all $\left.\eta^{\prime} \in\right] 0,1[$ and $0<\theta_{1}^{\prime}<\theta_{2}^{\prime}<\theta_{3}^{\prime}<\theta_{4}^{\prime}$, there exists $C^{\prime}$ such that

$$
\mathcal{H}\left(\eta^{\prime}, \theta_{1}^{\prime}, \theta_{2}^{\prime}, \theta_{3}^{\prime}, \theta_{4}^{\prime}, C^{\prime}\right)
$$

is true. Take two points $\left(t_{\ominus}, x_{\ominus}\right)$ and $\left(t_{\oplus}, x_{\oplus}\right)$ in $Q_{\ominus}^{\prime}$ and $Q_{\oplus}^{\prime}$. For $r$ big enough, there is a decomposition $x_{\ominus}=x_{0}, \ldots, x_{n}=x_{\oplus}$ and $t_{\ominus}=t_{0}<\cdots<t_{n}=t_{\oplus}$-where $n$ depends only on the $\eta, \eta^{\prime}, \theta_{i}$ and $\theta_{i}^{\prime}$ 's- such that we can obtain $u\left(t_{i}, x_{i}\right) \leq C u\left(t_{i+1}, x_{i+1}\right)$. So we can take $C^{\prime}=C^{n}$. For $r$ bounded, the condition $\Delta(\alpha)$ gives the inequality. For simplicity, we will denote

$$
\mathcal{H}\left(C_{\mathcal{H}}\right)=\mathcal{H}\left(0.99,0.01,0.1,0.11,100, C_{\mathcal{H}}\right) .
$$

These coefficients have been chosen for convenience when we apply this inequality (see typically Propositions 3.1 or 3.4 ). We will write $u\left(t_{\ominus}, x_{\ominus}\right) \leq C_{\mathcal{H}} u\left(t_{\oplus}, x_{\oplus}\right)$ as soon as $x_{\oplus}$ and $x_{\ominus}$ are in the contraction of a ball on which $u$ is a solution and "there is time" between $t_{\ominus}$ and $t_{\oplus}$ as well as before $t_{\ominus}$. We will not have to bother with technical coefficients if they don't exceed 10 .

Definition 1.6. Set $\eta \in] 0,1\left[\right.$ and $0<\theta_{1}<\theta_{2}<\theta_{3}<\theta_{4}$, $(\Gamma, \mu)$ satisfies the discrete-time parabolic Harnack inequality $H\left(\eta, \theta_{1}, \theta_{2}, \theta_{3}, \theta_{4}, C\right)$ if for all $x_{0} \in \Gamma, s \in \mathbb{R}, r \in \mathbb{R}^{+}$and every non-negative solution on $Q=\left(\mathbb{Z} \cap\left[s, s+\theta_{4} r^{2}\right]\right) \times B\left(x_{0}, r\right)$, we have

$$
\left(n_{\ominus}, x_{\ominus}\right) \in Q_{\ominus},\left(n_{\oplus}, x_{\oplus}\right) \in Q_{\oplus} \text { and } d\left(x_{\ominus}, x_{\oplus}\right) \leq n_{\oplus}-n_{\ominus}
$$


implies

$$
u\left(n_{\ominus}, x_{\ominus}\right) \leq C u\left(n_{\oplus}, x_{\oplus}\right),
$$

where $Q_{\ominus}=\left(\mathbb{Z} \cap\left[s+\theta_{1} r^{2}, s+\theta_{2} r^{2}\right]\right) \times B\left(x_{0}, \eta r\right)$ and $Q_{\oplus}=(\mathbb{Z} \cap[s+$ $\left.\left.\theta_{3} r^{2}, s+\theta_{4} r^{2}\right]\right) \times B\left(x_{0}, \eta r\right)$.

If the condition $d\left(x_{\ominus}, x_{\oplus}\right) \leq n_{\oplus}-n_{\ominus}$ is not satisfied, $u\left(n_{\ominus}, x_{\ominus}\right)$ has no influence on $u\left(n_{\oplus}, x_{\oplus}\right)$. It is always satisfied if $r \geq 2 \eta /\left(\theta_{3}-\theta_{2}\right)$ and in this case we can write

$$
\sup _{Q_{\ominus}} u \leq C \inf _{Q_{\oplus}} u
$$

The same remark as above holds for this inequality and we will denote

$$
H\left(C_{H}\right)=H\left(0.99,0.01,0.1,0.11,100, C_{H}\right) .
$$

\subsection{Statement of the results.}

Here is our main result:

Theorem 1.7. The three following properties are equivalent.

i) There exist $C_{1}, C_{2}, \alpha>0$ such that $D V\left(C_{1}\right), P\left(C_{2}\right)$ and $\Delta(\alpha)$ are true.

ii) There exists $C_{H}>0$ such that $H\left(C_{H}\right)$ is true.

iii) There exist $c_{l}, C_{l}, C_{r}, c_{r}>0$ such that $G\left(c_{l}, C_{l}, C_{r}, c_{r}\right)$ is true.

Theorem 3.8 states that i) implies iii), Theorem 3.10 that iii) implies ii) and Theorem 3.11 that ii) implies i).

The first part (i) implies iii) ) is the most difficult and an intermediate result is:

ii)' There exists $C_{\mathcal{H}}>0$ such that $\mathcal{H}\left(C_{\mathcal{H}}\right)$ is true,

which is proven in Section 2 by a Moser type iteration argument. In fact, ii)' is also equivalent to the three properties, since we can prove ii)' implies i) the same way we prove ii) implies i). In Section 3, we complete the proof. First ii)' implies estimates for $\mathcal{P}_{t}$, which yield estimates iii) for $p_{n}$ by comparison. Then iii) implies ii) and ii) implies i). In the last 
section, two properties about Hölder regularity and Green function are deduced for graphs which satisfy these properties.

Let us note that it is straightforward that ii) and iii) imply the hypothesis $\Delta(\alpha)$. For iii), just apply the lower bound to $p_{1}(x, y)$ where $y \in B(x, 1)$. And for ii), set $n=-0.5$ to obtain $p_{0}(x, x) \leq C_{H} p_{1}(x, y)$.

This result connects with [15] and [5] since in groups or in graphs with linear volume growth, Poincaré inequality is always satisfied. In Euclidian graphs $\mathbb{Z}^{n}$, the estimates which are well-known for uniform transitions (this is for instance a consequence of the result in groups) are here proved for non-uniform transitions ( $\mu_{x y}$ doesn't depend on $\left.y-x\right)$.

\subsection{Continuous or discrete time.}

To prove the Harnack inequality, we will use analytic methods which yield Cacciopoli inequalities. For these methods, the continuous time is naturally more convenient. We may have an idea of the problems if we look what happens on the two points graph $\Gamma=\{a, b\}$. Choose $p(a, a)=p(b, b)=\alpha$ and $p(a, b)=p(b, a)=1-\alpha$, this may be done if we set $\mu_{a a}=\mu_{b b}=\alpha$ and $\mu_{a b}=1-\alpha$. This gives

$$
\left\{\begin{array} { l } 
{ p _ { n } ( a , a ) = \frac { 1 + ( 2 \alpha - 1 ) ^ { n } } { 2 } , } \\
{ p _ { n } ( a , b ) = \frac { 1 - ( 2 \alpha - 1 ) ^ { n } } { 2 } , }
\end{array} \quad \left\{\begin{array}{l}
\mathcal{P}_{t}(a, a)=\frac{1+e^{(2 \alpha-2) t}}{2} \\
\mathcal{P}_{t}(a, b)=\frac{1-e^{(2 \alpha-2) t}}{2}
\end{array}\right.\right.
$$

Of course if $\alpha=1$, there is no link between the two points. Now, if $\alpha \neq 1, \mathcal{P}$ is always a simple relaxation $\left(\mathcal{P}_{t}(a, a)>\mathcal{P}_{t}(a, b)\right)$ whereas, for $\alpha<1 / 2, p$ is an oscillating relaxation or worse (for $\alpha=0$ ) a pure oscillation.

The first conclusion is that we have to force a minimum value on the diagonal of the Markov kernel if we want a discrete-time parabolic Harnack inequality or estimates from below. Indeed, they are not satisfied by this example for $\alpha=0$. This has nothing to do with the fact that the graph is finite, take the standard random walk on $\mathbb{Z}$ and observe its effect on $u(0, z)=z \bmod 2$. We obtain $u(n, z)=(n+z) \bmod 2$.

What plays a role is condition $\Delta(\alpha)$ and particulary the fact that $p(x, x) \geq \alpha$. We have extended this condition to $p(x, y) \geq \alpha$ for $x \sim y$ so that our results are true for low values of $n$, think for instance to the lower bound of $p_{1}(x, y)=p(x, y)$. Besides, lower bounds for $d(x, y)=n$ 
are the consequence of lower bounds for $n=1$, see the proof of Theorem 3.8 .

The second conclusion is that the behaviour of the discrete-time Markov chain is more difficult to control. In addition to the usual heat relaxation, there may be another phenomenon of relaxation of the oscillating errors due to the discretization of the time.

One more attempt to show the difficulty of adapting the analytic methods to the discrete time. Consider the proof of the Cacciopoli inequality. When time and space are continuous, take $u$ such that $\partial u / \partial t=\triangle u$ and a compactly supported cut-off function $\psi$ to integrate by parts.

$$
\begin{aligned}
\frac{1}{2} \iint \psi^{2} \frac{\partial\left(u^{2}\right)}{\partial t} & =\iint \psi^{2} u \triangle u \\
& =-\iint \nabla\left(\psi^{2} u\right) \cdot \nabla u \\
& =-\iint \psi^{2}|\nabla u|^{2}-\iint 2 \psi u \nabla \psi \cdot \nabla u
\end{aligned}
$$

Since

$$
-\iint 2 \psi \nabla \psi u \nabla u \leq \frac{1}{2} \iint \psi^{2}|\nabla u|^{2}+2 \iint|\nabla \psi|^{2} u^{2}
$$

one gets

$$
\frac{1}{2} \iint \psi^{2} \frac{\partial\left(u^{2}\right)}{\partial t}+\frac{1}{2} \iint \psi^{2}|\nabla u|^{2} \leq 2 \iint|\nabla \psi|^{2} u^{2}
$$

This inequality is essential to estimate $\|\nabla u\|_{2}$ with $\|u\|_{2}$, which with the Sobolev inequality gives estimates between mean values for exponents of the same sign. Let us try to adapt this argument to discrete time. Note that (1.2) may be written in the following way

$$
m(x)(u(n+1, x)-u(n, x))=\sum \mu_{x y}(u(n, y)-u(n, x)) .
$$

For simplicity, we will forget about the cut-off function (take $u(n, \cdot)$ 
compactly supported). Write

$$
\begin{aligned}
2 \sum_{x} m(x) u( & n, x)(u(n+1, x)-u(n, x)) \\
= & 2 \sum_{x, y} \mu_{x y} u(n, x)(u(n, y)-u(n, x)) \\
= & \sum_{x, y} \mu_{x y} u(n, x)(u(n, y)-u(n, x)) \\
& +\sum_{x, y} \mu_{y x} u(n, y)(u(n, x)-u(n, y)) \\
= & -\sum_{x, y} \mu_{x y}(u(n, y)-u(n, x))^{2} .
\end{aligned}
$$

This is nice but we have not taken the exact time differentiation of $\sum_{x} m(x) u^{2}(n, x)$, that is,

$$
\begin{aligned}
\sum_{x} m(x)\left(u^{2}(n+1, x)\right. & \left.-u^{2}(n, x)\right) \\
= & 2 \sum_{x} m(x) u(n, x)(u(n+1, x)-u(n, x)) \\
& +\sum_{x} m(x)(u(n+1, x)-u(n, x))^{2} \\
= & -\sum_{x, y} \mu_{x y}(u(n, y)-u(n, x))^{2} \\
& +\sum_{x} m(x)(u(n+1, x)-u(n, x))^{2} .
\end{aligned}
$$

Fortunately, if we suppose that $\mu_{x x} \geq \alpha m(x)$, then

$$
\begin{aligned}
\sum_{x} m(x)(u(n & +1, x)-u(n, x))^{2} \\
& =\sum_{x} \frac{1}{m(x)}\left(\sum_{y} \mu_{x y}(u(n, y)-u(n, x))\right)^{2} \\
& \leq \sum_{x} \frac{1}{m(x)}\left(\sum_{y \neq x} \mu_{x y}\right)\left(\sum_{y} \mu_{x y}(u(n, y)-u(n, x))^{2}\right) \\
& \leq(1-\alpha) \sum_{x, y} \mu_{x y}(u(n, y)-u(n, x))^{2} .
\end{aligned}
$$


This yields

$$
\sum_{x} m(x)\left(u^{2}(n+1, x)-u^{2}(n, x)\right) \leq-\alpha \sum_{x, y} \mu_{x y}(u(n, y)-u(n, x))^{2}
$$

The constant $\alpha$ has been used to control the errors due to the discrete time. But these manipulations seem far more intricate when we deal with subsolutions or the logarithm of $u$ (and cut-off functions). Therefore, we won't try to apply Moser's iterative technique directly to solutions of the discrete-time parabolic equation.

\section{Harnack inequality for solutions of the continuous-time parabolic equation.}

Theorem 2.1. Assume $(\Gamma, \mu)$ satisfies $D V\left(C_{1}\right), P\left(C_{2}\right)$ and $\Delta(\alpha)$. Then, there exists $C_{\mathcal{H}}$ such that $\mathcal{H}\left(C_{\mathcal{H}}\right)$ is true.

The proof is an adaptation of [30]. The strategy is Moser's iterative technique [22], that is to prove inequalities involving the mean values

$\mathcal{M}\left(u, p,\left[s_{1}, s_{2}\right] \times B\right)=\left(\frac{1}{\left(s_{2}-s_{1}\right) V(B)} \sum_{x \in B} \int_{s_{1}}^{s_{2}} m(x) u^{2 p}(t, x) d t\right)^{1 / p}$

The idea is we get the infimum when $p \longrightarrow-\infty$ and the supremum when $p \longrightarrow+\infty$. Thus we want to prove a series of inequalities between $-\infty$ and $+\infty$. To improve the exponent of a mean value, the Sobolev inequality proved in Section 2.1 is helpful. One application of this inequality yields an elementary step of the iterative technique proved in Section 2.2. The iteration gives inequalities between the extrema and mean values as stated in Section 2.3. The most difficult step is between negative and positive values. Here we use an improvement of the initial version [22] proposed in [23] with an idea of E. Bombieri [2]. This is the object of Section 2.4 and needs a weighted Poincaré inequality stated in Section 2.1.

Throughout this section devoted to the proof of Theorem 2.1, $D V\left(C_{1}\right), P\left(C_{2}\right)$ and $\Delta(\alpha)$ are assumed and $u>0$. The theorem for $u \geq 0$ is then straightforward. 


\subsection{Poincaré and Sobolev inequalities.}

Proposition 2.2 (Weighted Poincaré inequality). There exists $C$ depending on $C_{1}, C_{2}$ and $\alpha$ such that for all $x_{0} \in \Gamma, R \in \mathbb{N}$ and $f \in$ $\mathbb{R}^{B\left(x_{0}, R\right)}$,

$$
\begin{aligned}
& \sum_{x \in B\left(x_{0}, R\right)} m(x) \psi^{2}(x)\left(f(x)-f_{B \psi}\right)^{2} \\
& \leq C R^{2} \sum_{x, y \in B\left(x_{0}, R\right)} \mu_{x y} \min \left\{\psi^{2}(x), \psi^{2}(y)\right\}(f(y)-f(x))^{2},
\end{aligned}
$$

where $\psi(x)=1-d\left(x_{0}, x\right) / R$ and $f_{B \psi}$ is such that the term on the left is minimal, that is

$$
f_{B \psi}=\frac{\sum_{x \in B\left(x_{0}, R\right)} m(x) \psi^{2}(x) f(x)}{\sum_{x \in B\left(x_{0}, R\right)} m(x) \psi^{2}(x)}
$$

Proof. We refer to the proof in [32] based on [17]. Consider $\mathcal{F}$ a collection of balls with the following tree structure: denote one ball $B_{1}$ -the root of the tree- and assume that there is a function $B \longmapsto \bar{B}$ from $\mathcal{F} \backslash\left\{B_{1}\right\}$ to $\mathcal{F}$ (denote $B^{(i)}$ its iteration) such that for all $B \in \mathcal{F}$, $\operatorname{rg} B=\inf \left\{k: B^{(k-1)}=B_{1}\right\}<\infty$. Denote $r(B)$ the radius of $B$, $A[B]=\left\{\tilde{B} \in \mathcal{F}:\right.$ exists $\left.k \in \mathbb{N}, \tilde{B}^{(k)}=B\right\}$ and $B^{*}=1.001 B$. For our discrete setting, we will need this version of Poincaré inequality where $C_{2}^{\prime}$ depends on $C_{1}, C_{2}$ and $\alpha$.

$$
\sum_{x \in B\left(x_{0}, r\right)} m(x)\left|f(x)-f_{B}\right|^{2} \leq C_{2}^{\prime} r^{2} \sum_{x, y \in B\left(x_{0}, 1.001 r\right)} \mu_{x y}(f(y)-f(x))^{2},
$$

for all $f \in \mathbb{R}^{\Gamma}$, for all $x_{0} \in \Gamma$, for all $r \in \mathbb{R}^{+}$. It is obtained by an easy covering argument. Again, there may be some problems for small $r$ but then $\Delta(\alpha)$ gives the inequality.

The following lemma will be applied to

$$
\mu_{x y}^{\prime}=\mu_{x y} \min \left\{\psi^{2}(x), \psi^{2}(y)\right\} .
$$

The notations $m^{\prime}$ or $f_{B}^{\prime}$ should be understood with respect to $\mu^{\prime}$. 
Lemma 2.3. Assume there exists $C$ such that, for all $B \in \mathcal{F}$, there exists $c_{B}$ such that

$$
\begin{aligned}
& \begin{cases}\frac{c_{B}}{C} m(x) \leq m^{\prime}(x) \leq C c_{B} m(x), & \text { for all } x \in B^{*}, \\
\frac{c_{B}}{C} \mu_{x y} \leq \mu_{x y}^{\prime} \leq C c_{B} \mu_{x y}, & \text { for all } x, y \in B^{*}\end{cases} \\
& \#\left\{B \in \mathcal{F}: x \in B^{*}\right\} \leq C, \quad \text { for all } x \in \Gamma, \\
& \mu(B \cap \bar{B}) \geq \frac{\max \{\mu(B), \mu(\bar{B})\}}{C}, \quad \text { for all } B \in \mathcal{F} \text {. }
\end{aligned}
$$

Then for every function $f$,

$$
\begin{gathered}
\sum_{x \in \cup_{B \in \mathcal{F}} B} m^{\prime}(x)\left(f(x)-f_{B_{1}}^{\prime}\right)^{2} \leq 4 C_{2}^{\prime} C^{8} \sup _{B \in \mathcal{F}}\left(r^{2}(B) \sum_{\tilde{B} \in A[B]} \frac{\mu(\tilde{B})}{\mu(B)} \operatorname{rg} \tilde{B}\right) \\
\cdot \sum_{x, y \in \cup_{B \in \mathcal{F}} B^{*}} \mu_{x y}^{\prime}(f(x)-f(y))^{2} .
\end{gathered}
$$

Proof. In fact condition (2.7) is needed for $\mu^{\prime}$ but because of (2.5), (2.7) implies that

$$
\mu^{\prime}(B \cap \bar{B}) \geq \frac{\max \left\{\mu^{\prime}(B), \mu^{\prime}(\bar{B})\right\}}{C^{3}}, \quad \text { for all } B \in \mathcal{F} .
$$

First note that

$$
\begin{aligned}
& \left(f_{B}^{\prime}-f_{\bar{B}}^{\prime}\right)^{2} \\
& =\frac{\sum_{x \in B \cap \bar{B}} m^{\prime}(x)\left(\left(f_{B}^{\prime}-f(x)\right)+\left(f(x)-f_{\bar{B}}^{\prime}\right)\right)^{2}}{\mu^{\prime}(B \cap \bar{B})} \\
& \leq \frac{2}{\mu^{\prime}(B \cap \bar{B})}\left(\sum_{x \in B} m^{\prime}(x)\left(f(x)-f_{B}^{\prime}\right)^{2}+\sum_{x \in \bar{B}} m^{\prime}(x)\left(f(x)-f_{\bar{B}}^{\prime}\right)^{2}\right) .
\end{aligned}
$$

These terms $\sum_{x \in B} m^{\prime}(x)\left(f(x)-f_{B}^{\prime}\right)^{2}$ satisfy

$$
\sum_{x \in B} m^{\prime}(x)\left(f(x)-f_{B}^{\prime}\right)^{2} \leq \sum_{x \in B} m^{\prime}(x)\left(f(x)-f_{B}\right)^{2}
$$




$$
\begin{aligned}
& \leq C c_{B} \sum_{x \in B} m(x)\left(f(x)-f_{B}\right)^{2} \\
& \leq C c_{B} C_{2}^{\prime} r^{2}(B) \sum_{x, y \in B^{*}} \mu_{x y}(f(x)-f(y))^{2} \\
& \leq C^{2} C_{2}^{\prime} r^{2}(B) \sum_{x, y \in B^{*}} \mu_{x y}^{\prime}(f(x)-f(y))^{2}
\end{aligned}
$$

We can now prove the lemma,

$$
\begin{aligned}
& \sum_{x \in \cup_{B \in \mathcal{F}} B} m^{\prime}(x)\left(f(x)-f_{B_{1}}^{\prime}\right)^{2} \\
& \leq \sum_{B \in \mathcal{F}} \sum_{x \in B} m^{\prime}(x) \operatorname{rg} B\left(\left(f(x)-f_{B}^{\prime}\right)^{2}+\sum_{i=1}^{\operatorname{rg} B-1}\left(f_{B^{(i-1)}}^{\prime}-f_{B^{(i)}}^{\prime}\right)^{2}\right) \\
& \leq \sum_{B \in \mathcal{F}}\left(\sum_{\tilde{B} \in A[B]} 2 \mu^{\prime}(\tilde{B}) \operatorname{rg} \tilde{B} \frac{2}{\mu^{\prime}(B) / C^{3}}\right) \sum_{x \in B} m^{\prime}(x)\left(f(x)-f_{B}^{\prime}\right)^{2} \\
& \leq 4 C^{3} \sum_{B \in \mathcal{F}}\left(\sum_{\tilde{B} \in A[B]} \frac{\mu^{\prime}(\tilde{B})}{\mu^{\prime}(B)} \operatorname{rg} \tilde{B}\right) C^{2} C_{2}^{\prime} r^{2}(B) \sum_{x, y \in B^{*}} \mu_{x y}^{\prime}(f(x)-f(y))^{2} \\
& \leq 4 C_{2}^{\prime} C^{5} \sum_{x, y \in \cup_{B \in \mathcal{F}} B^{*}} C_{B \in \mathcal{F}}\left(r^{2}(B) \sum_{\tilde{B} \in A[B]} \frac{\mu^{\prime}(\tilde{B})}{\mu^{\prime}(B)} \operatorname{rg} \tilde{B}\right) \\
& \cdot \mu_{x y}^{\prime}(f(x)-f(y))^{2} .
\end{aligned}
$$

To finish the proof, we replace $\mu^{\prime}(\tilde{B})$ and $\mu^{\prime}(B)$ by $\mu(\tilde{B})$ and $\mu(B)$ so that another factor $C^{2}$ appears.

END OF PROOF OF Proposition 2.2. We will construct $\mathcal{F}$ as a Whitney covering of $B\left(x_{0}, R-1\right)$ by selecting $W_{n} \subset\left\{x: d\left(x_{0}, x\right)=R-2^{n}\right\}$ for $0 \leq n \leq N=[\log R / \log 2]$.

$$
\mathcal{F}=\left\{B(x, r): \text { exists } n, x \in W_{n} \text { and } r=\frac{2^{n}}{1.01}\right\} \cup\left\{B_{1}\right\},
$$

where $B_{1}=B\left(x_{0}, R / 1.01\right)$. For these balls, $(2.5)$ is satisfied. The tree structure will be constructed this way: if $B=B(x, r)$ with $x \in W_{n}$ we will choose $\bar{B}$ of center $\bar{x} \in W_{n+1}$ such that $d(x, \bar{x}) \leq(3 / 2) 2^{n}$ (see the construction of the $W_{n}$ 's below). Thus, $B\left(x,(2 / 1.01-3 / 2) 2^{n}\right) \subset B \cap \bar{B}$ and condition (2.7) is satisfied. 
We must check, in order to apply Lemma 2.3, that it was possible to select $W_{n}$ so that

$$
\left\{x: R-2^{n+1} \leq d\left(x_{0}, x\right) \leq R-2^{n}\right\} \subset \bigcup_{x \in W_{n+1}} B\left(x, \frac{3}{2} 2^{n}\right),
$$

while (2.6) is satisfied. It is a standard Besicovitch covering argument, we choose a minimal $W_{n+1}$ for this property. The key is that the radius of any ball $B^{*}$ such that $x \in B^{*}$ is comparable to $R-d\left(x_{0}, x\right)$.

Now let us consider the term

$$
\sup _{B \in \mathcal{F}}\left(r^{2}(B) \sum_{\tilde{B} \in A[B]} \frac{\mu(\tilde{B})}{\mu(B)} \operatorname{rg} \tilde{B}\right) .
$$

The first point is that $d(x, \bar{x}) \leq(3 / 2) 2^{n}$ implies that $2 B \subset 2 \bar{B}$. For $B \in \mathcal{F} \backslash\left\{B_{1}\right\}$, set $n \leq N$ such that $W_{n}$ contains $B$ 's center. If we denote $\mathcal{F}_{k}=\left\{\tilde{B}: \tilde{B}^{(k)}=B\right\}$ and $A_{k}=\bigcup_{\tilde{B} \in \mathcal{F}_{k}} 2 \tilde{B}, A_{k+1} \subset A_{k}$. But there is more than this inclusion, a ball $\tilde{B}=B(\tilde{x}, \tilde{r})$ in $\mathcal{F}_{k}$ is such that $d\left(x_{0}, \tilde{x}\right)=R-2^{n-k}$ and $\tilde{r}=2^{n-k} / 1.01$, so that there is a ball of radius $\tilde{r} / 100$ which is included in $2 \tilde{B}$ and in the area $\{y$ : $\left.d\left(x_{0}, y\right)<R-2^{n-1-k}-2 \cdot 2^{n-1-k} / 1.01\right\}$ never reached by $A_{k+1}$. This yields $\mu\left(A_{k} \backslash A_{k+1}\right) \geq \varepsilon \mu\left(A_{k}\right)$ and consequently $\mu\left(A_{k}\right) \leq e^{-c k} \mu\left(A_{0}\right) \leq$ $C e^{-c k} \mu(B)$. Thus,

$r^{2}(B) \sum_{\tilde{B} \in A[B]} \frac{\mu(\tilde{B})}{\mu(B)} \operatorname{rg} \tilde{B} \leq 2^{2 n} \sum_{k \geq 0} C e^{-c k}(N+1-n+k) \leq C 2^{2 N} \leq C R^{2}$.

For the case $B=B_{1}$, the proof is identical but we refer to $A_{1}$ instead of $A_{0}$.

To finish the proof, let us compare $m(x) \psi^{2}(x)$ and $m^{\prime}(x)$. For $x \neq x_{0}$, the condition $\Delta(\alpha)$ gives $m(x) \psi^{2}(x) \leq m^{\prime}(x) / \alpha$, we just have to consider $y \sim x$ such that $d\left(x_{0}, x\right)=d\left(x_{0}, y\right)+1$.

Proposition 2.4 (Sobolev-Poincaré inequality). There exist $\theta>1$ depending on $C_{1}$ and $S$ depending on $C_{1}, C_{2}$ and $\alpha$ such that for every function $f$ on $B$ of radius $r$,

$$
\begin{aligned}
& \left(\frac{1}{V(B)} \sum_{x \in B} m(x) f^{2 \theta}(x)\right)^{1 / \theta} \\
& \quad \leq \frac{S}{V(B)}\left(r^{2} \sum_{x, y \in B} \mu_{x y}(f(y)-f(x))^{2}+\sum_{x \in B} m(x) f^{2}(x)\right) .
\end{aligned}
$$


In the setting of manifolds, this result which was proven in [29] was the key of the proof of the Harnack inequality after the work [28]. It is adapted to graphs in [9]. A nice abstract version can also be found in [14]. In the notation of this paper, the chain condition will be satisfied for $\lambda<2$ (like the preceding section where $\lambda=1.001$ ). Indeed, consider $x$ next to the boundary of $B$, the smallest ball $B_{i}$ of the chain not centered at $x$ must contain $x$ and satisfy $\lambda B_{i} \subset B$.

\subsection{Elementary step of Moser's iterative technique.}

As in Section 1.3, we will say that $u$ is a positive sub/supersolution on $Q=I \times B\left(x_{0}, r\right)$ if it is the trace of a positive function on $I \times B\left(x_{0}, r+\right.$ 1 ) which is a sub/supersolution everywhere on $Q$. Precisely, we say that $u$ is a positive subsolution on $Q$ if it is positive and

$$
m(x) \frac{\partial}{\partial t} u(t, x) \leq \sum_{y} \mu_{x y}(u(t, y)-u(t, x)),
$$

for all $t \in I$, for all $x \in B\left(x_{0}, r-1\right)$. And $u$ is a positive supersolution on $Q$ if it is positive and

$$
m(x) \frac{\partial}{\partial t} u(t, x) \geq \sum_{y} \mu_{x y}(u(t, y)-u(t, x)),
$$

for all $t \in I$, for all $x \in B\left(x_{0}, r-1\right)$,

$$
m(x)\left(\frac{\partial}{\partial t} u(t, x)+u(t, x)\right) \geq \sum_{y \in B\left(x_{0}, r\right)} \mu_{x y} u(t, y),
$$

for all $t \in I$, for all $x$ such that $d\left(x_{0}, x\right)=[r]$. Let us show the elementary step of Moser's iterative technique. If $Q=I \times B$ where $I=\left[s_{1}, s_{2}\right]$ and $B=B(x, r)$, note

$$
\begin{gathered}
B_{\sigma}=(1-\sigma) B=B(x,(1-\sigma) r), \\
I_{\sigma}=\left[\left(1-\sigma^{2}\right) s_{1}+\sigma^{2} s_{2}, s_{2}\right], \\
I_{\sigma}^{\prime}=\left[s_{1}, \sigma^{2} s_{1}+\left(1-\sigma^{2}\right) s_{2}\right], \\
I_{\sigma}^{\prime \prime}=\left[\left(1-\sigma^{2}\right) s_{1}+\sigma^{2} s_{2}, \sigma^{2} s_{1}+\left(1-\sigma^{2}\right) s_{2}\right], \\
Q_{\sigma}=I_{\sigma} \times B_{\sigma}, Q_{\sigma}^{\prime}=I_{\sigma}^{\prime} \times B_{\sigma} \text { and } Q_{\sigma}^{\prime \prime}=I_{\sigma}^{\prime \prime} \times B_{\sigma} .
\end{gathered}
$$


Note that

$$
Q_{\sigma_{1}+\sigma_{2}} \subset\left(Q_{\sigma_{1}}\right)_{\sigma_{2}}, Q_{\sigma_{1}+\sigma_{2}}^{\prime} \subset\left(Q_{\sigma_{1}}^{\prime}\right)_{\sigma_{2}}^{\prime} \text { and } Q_{\sigma_{1}+\sigma_{2}}^{\prime \prime} \subset\left(Q_{\sigma_{1}}^{\prime \prime}\right)_{\sigma_{2}}^{\prime \prime}
$$

Lemma 2.5. There is an exponent $\kappa=2-1 / \theta$ and a constant $A=$ $A\left(C_{1}, S\right)=A\left(C_{1}, C_{2}, \alpha\right)$ such that if $B=B\left(x_{0}, r\right), Q=\left[0, r^{2}\right] \times B, u$ a positive subsolution in $Q$ and $1 / r \leq \sigma \leq 1 / 2$, then

$$
\mathcal{M}\left(u, \kappa, Q_{\sigma}\right) \leq\left(\frac{A}{\sigma^{2}}\right)^{1 / \kappa} \mathcal{M}(u, 1, Q) .
$$

If $u$ is a supersolution with the same assumptions, then

$$
\mathcal{M}\left(u, \kappa, Q_{\sigma}^{\prime}\right) \leq\left(\frac{A}{\sigma^{2}}\right)^{1 / \kappa} \mathcal{M}(u, 1, Q) .
$$

Proof. Consider the first part, $u$ is a subsolution. Let $\psi$ be a nonnegative function in $B$, with $d\left(x_{0}, x\right)=r$ implies $\psi(x)=0$, then

$$
\begin{aligned}
& \sum_{x \in B} m(x) \psi^{2}(x) u(t, x) \frac{\partial}{\partial t} u(t, x) \\
& \leq \sum_{x, y \in B} \mu_{x y} \psi^{2}(x) u(t, x)(u(t, y)-u(t, x)) \\
& =\frac{1}{2} \sum_{x, y \in B} \mu_{x y}\left(\psi^{2}(x) u(t, x)-\psi^{2}(y) u(t, y)\right)(u(t, y)-u(t, x)) \\
& =\frac{1}{2} \sum_{x, y \in B} \mu_{x y} \psi^{2}(x)(u(t, x)-u(t, y))(u(t, y)-u(t, x)) \\
& \quad+\frac{1}{2} \sum_{x, y \in B} \mu_{x y}\left(\psi^{2}(x)-\psi^{2}(y)\right) u(t, y)(u(t, y)-u(t, x)) .
\end{aligned}
$$

In the last term, we use the inequality $a b \leq a^{2} / 4+b^{2}$.

$$
\begin{aligned}
\left(\psi^{2}(x)-\psi^{2}(y)\right) u(t, y)( & u(t, y)-u(t, x)) \\
= & \psi(x)(\psi(x)-\psi(y)) u(t, y)(u(t, y)-u(t, x)) \\
& +\psi(y)(\psi(x)-\psi(y)) u(t, y)(u(t, y)-u(t, x)) \\
\leq & \frac{1}{4}\left(\psi^{2}(x)+\psi^{2}(y)\right)(u(t, y)-u(t, x))^{2} \\
& +2 u^{2}(t, y)(\psi(x)-\psi(y))^{2}
\end{aligned}
$$


Note that because of the symmetry of the weights $\mu_{x y}$,

$\sum_{x, y \in B} \mu_{x y} \psi^{2}(y)(u(t, y)-u(t, x))^{2}=\sum_{x, y \in B} \mu_{x y} \psi^{2}(x)(u(t, y)-u(t, x))^{2}$.

Thus, (2.9) yields

$$
\begin{aligned}
\sum_{x \in B} m(x) \psi^{2}(x) u(t, x) \frac{\partial}{\partial t} u(t, x)+ & \frac{1}{4} \sum_{x, y \in B} \mu_{x y} \psi^{2}(x)(u(t, y)-u(t, x))^{2} \\
& \leq \sum_{x, y \in B} \mu_{x y} u^{2}(t, y)(\psi(x)-\psi(y))^{2}
\end{aligned}
$$

For $u$ supersolution, the result would be

$$
\begin{aligned}
& \sum_{x \in B} m(x) \psi^{2}(x) u(t, x) \frac{-\partial}{\partial t} u(t, x) \\
& +\frac{1}{4} \sum_{x, y \in B} \mu_{x y} \psi^{2}(x)(u(t, y)-u(t, x))^{2} \\
& \quad \leq \sum_{x, y \in B} \mu_{x y} u^{2}(t, y)(\psi(x)-\psi(y))^{2}
\end{aligned}
$$

And then, the same arguments work dealing with $I_{\sigma}^{\prime}$ instead of $I_{\sigma}$. Return now to (2.10), if $\chi$ is a smooth function of $t$, we obtain

$$
\begin{gathered}
\frac{\partial}{\partial t}\left(\sum_{x \in B} m(x)(\chi(t) \psi(x) u(t, x))^{2}\right) \\
+\frac{\chi^{2}(t)}{2} \sum_{x, y \in B} \mu_{x y} \psi^{2}(x)(u(t, y)-u(t, x))^{2} \\
\leq 2 \chi^{2}(t) \sum_{x, y \in B} \mu_{x y} u^{2}(t, y)(\psi(x)-\psi(y))^{2} \\
+\sum_{x \in B} m(x)\left(\frac{\partial}{\partial t} \chi^{2}(t)\right) u^{2}(t, x) .
\end{gathered}
$$

Now we choose $\chi(t)=t /(\sigma r)^{2} \wedge 1$ and $\psi$ so that $d\left(x_{0}, x\right)=r$ implies $\psi(x)=0$ and $\psi \equiv 1$ in $B_{\sigma}$. For this purpose, we took $1 / r \leq \sigma$. 
Integrating over $I$ yields

$$
\left\{\begin{array}{c}
\sup _{t \in I_{\sigma}}\left(\sum_{x \in B_{\sigma}} m(x) u^{2}(t, x)\right) \leq \frac{10}{(\sigma r)^{2}} \int_{I} \sum_{x \in B} m(x) u^{2}(t, x) d t \\
\frac{1}{2} \int_{I_{\sigma}} \sum_{x, y \in B_{\sigma}} \mu_{x y}(u(t, y)-u(t, x))^{2} d t \\
\leq \frac{10}{(\sigma r)^{2}} \int_{I} \sum_{x \in B} m(x) u^{2}(t, x) d t
\end{array}\right.
$$

We have used $\left|\chi^{\prime}\right| \leq 1 /(\sigma r)^{2}$ and $|\psi(x)-\psi(y)| \leq 2 /(\sigma r)$ when $x \sim y$. This result (of Cacciopoli type) allows us to use Proposition 2.4 (Sobolev). Note $\theta^{\prime}$ such that $1 / \theta+1 / \theta^{\prime}=1$ and $\kappa=1+1 / \theta^{\prime}$.

$$
\begin{aligned}
\mathcal{M}\left(u, \kappa, Q_{\sigma}\right)^{\kappa}= & \frac{1}{V\left(B_{\sigma}\right) r^{2}\left(1-\sigma^{2}\right)} \int_{I_{\sigma}} \sum_{x \in B_{\sigma}} m(x) u^{2 \kappa}(t, x) d t \\
\leq & \frac{1}{r^{2}\left(1-\sigma^{2}\right)} \int_{I_{\sigma}}\left(\frac{1}{V\left(B_{\sigma}\right)} \sum_{x \in B_{\sigma}} m(x) u^{2}(t, x)\right)^{1 / \theta^{\prime}} \\
& \cdot\left(\frac{1}{V\left(B_{\sigma}\right)} \sum_{x \in B_{\sigma}} m(x) u^{2 \theta}(t, x)\right)^{1 / \theta} d t \\
\leq & \frac{\sup _{t \in I_{\sigma}}\left(\sum_{x \in B_{\sigma}} m(x) u^{2}(t, x)\right)^{1 / \theta^{\prime}}}{r^{2}\left(1-\sigma^{2}\right) V\left(B_{\sigma}\right)^{1 / \theta^{\prime}}} \\
& \cdot \int_{I_{\sigma}} \frac{S}{V\left(B_{\sigma}\right)}\left(r^{2} \sum_{x, y \in B_{\sigma}} \mu_{x y}(u(t, y)-u(t, x))^{2}\right. \\
\leq & \frac{1}{r^{2}\left(1-\sigma^{2}\right)}\left(\frac{10}{(\sigma r)^{2}}\right)^{1 / \theta^{\prime}}\left(\frac{20}{\sigma^{2}}+1\right) \frac{S}{V\left(B_{\sigma}\right)^{1 / \theta^{\prime}+1}} \\
& \cdot\left(\int_{I} \sum_{x \in B} m(x) u^{2}(t, x) d t\right)^{1 / \theta^{\prime}+1} \cdot \\
&
\end{aligned}
$$

This yields

$$
\mathcal{M}\left(u, \kappa, Q_{\sigma}\right) \leq\left(\frac{A}{\sigma^{2}}\right)^{1 / \kappa} \mathcal{M}(u, 1, Q)
$$


for a constant $A$, because $\sigma \leq 1 / 2$ so that $V(B) \leq C_{1} V\left(B_{\sigma}\right)$ and $1-\sigma^{2} \geq 3 / 4$.

\subsection{Mean value inequalities.}

Lemma 2.6. If $u$ is a positive solution on $I \times B$, then

- $u^{p}$ is a subsolution on $I \times B$ for $p \leq 0$ and $p \geq 1$,

- $u^{p}$ is a supersolution on $I \times B$ for $0 \leq p \leq 1$.

ProOF. Let $f(x)=x^{p}$, if $p \leq 0$ or $p \geq 1, f$ is convex and

$$
f^{\prime}(a)(b-a) \leq f(b)-f(a)
$$

This yields

$$
\begin{aligned}
m(x) \frac{\partial}{\partial t} f(u(t, x)) & =m(x) f^{\prime}(u(t, x)) \frac{\partial}{\partial t} u(t, x) \\
& =\sum_{y} \mu_{x y} f^{\prime}(u(t, x))(u(t, y)-u(t, x)) \\
& \leq \sum_{y} \mu_{x y}(f(u(t, y))-f(u(t, x))) .
\end{aligned}
$$

Lemma 2.7. Let $B$ be a ball of radius $r, Q=\left[0, r^{2}\right] \times B, u$ a positive solution on $Q$ and $0<\delta \leq 1 / 2$. Then, for all $p>0$,

$$
\begin{aligned}
& \mathcal{M}(u,-p, Q) \leq C\left(C \delta^{-\gamma}\right)^{1 / p} \inf _{Q_{\delta}} u^{2}, \\
& \sup _{Q_{\delta}^{\prime \prime}} u^{2} \leq C\left(C \delta^{-\gamma}\right)^{1 / p} \mathcal{M}(u, p, Q),
\end{aligned}
$$

where $C$ and $\gamma$ depend only on $C_{1}, C_{2}$ and $\alpha$.

Proof. We will prove (2.11). Consider first the case $\delta r<2$ (the difference between $B$ 's radius and $B_{\delta}$ 's is less than 2 , this includes the cases $B=B_{\delta}$ when one can not apply the elementary step, Lemma 2.5). Take $u^{2}(t, z)=\inf _{Q_{\delta}} u^{2}$ and note that, for all $0 \leq \tau \leq \delta^{2} r^{2}, t-\tau \in I$ 
and $u^{2}(t-\tau, z) \leq e^{2 \tau} u^{2}(t, z) \leq e^{8} \inf _{Q_{\delta}} u^{2}$. This is a consequence of (1.4). Counting only these values,

$$
\mathcal{M}(u,-p, Q)^{-p} \geq \frac{m(z) \delta^{2} r^{2}}{r^{2} V(B)}\left(e^{8} \inf _{Q_{\delta}} u^{2}\right)^{-p}
$$

implies

$$
\mathcal{M}(u,-p, Q) \leq e^{8}\left(\frac{V(B)}{V(z, 1 / 2) \delta^{2}}\right)^{1 / p} \inf _{Q_{\delta}} u^{2} .
$$

Applying first $D V\left(C_{1}\right)$ between $B(z, 2 r) \supset B$ and $B(z, 1 / 2)$ then $r<$ $2 \delta^{-1}$, we obtain $(2.11)$.

Consider now $\delta r \geq 2$. Set $\sigma_{i}=2^{-i} \delta, Q(0)=Q$ and $Q(i)=$ $Q(i-1)_{\sigma_{i}}$ so that for all $i, Q_{\delta} \subset Q(i)$. Fix $n$ the integer such that $2^{n+1} \leq \delta r<2^{n+2}$. We can apply Lemma 2.5 between $Q(i-1)$ and $Q(i)$ for $i \leq n$ since $u^{-q}$ is a subsolution, the radius of the cylinder $Q(i-1)$ is bigger than $r / 2$ and $\sigma_{i} \geq 2 / r$.

$$
\mathcal{M}(u,-q \kappa, Q(i))^{-1 / q} \leq\left(\frac{A}{\sigma_{i}^{2}}\right)^{1 / \kappa} \mathcal{M}(u,-q, Q(i-1))^{-1 / q}
$$

implies

$$
\mathcal{M}(u,-q, Q(i-1)) \leq\left(\frac{A}{\sigma_{i}^{2}}\right)^{1 /(q \kappa)} \mathcal{M}(u,-q \kappa, Q(i)) .
$$

This yields

$$
\mathcal{M}(u,-p, Q) \leq\left(\prod_{i=1}^{n}\left(\frac{A}{\left(2^{-i} \delta\right)^{2}}\right)^{1 / \kappa^{i}}\right)^{1 / p} \mathcal{M}\left(u,-p \kappa^{n}, Q(n)\right) .
$$

To obtain (2.11), we may first check that

$$
\prod_{i=1}^{+\infty}\left(\frac{A}{\left(2^{-i} \delta\right)^{2}}\right)^{1 / \kappa^{i}} \leq C \delta^{-\gamma}
$$

Then, we estimate $\mathcal{M}\left(u,-p \kappa^{n}, Q(n)\right)$ as in the case $\delta r<2$. Take $u^{2}(t, z)=\inf _{Q(n+1)} u^{2}$ and note that for all $0 \leq \tau \leq\left(\sigma_{n+1} r / 2\right)^{2}$, $t-\tau \in I(n)$ and $u^{2}(t-\tau, z) \leq e^{2 \tau} u^{2}(t, z) \leq e^{2} \inf _{Q(n+1)} u^{2}$. We use $1 \leq \sigma_{n+1} r<2$. This yields

$$
\mathcal{M}\left(u,-p \kappa^{n}, Q(n)\right) \leq e^{2}\left(\frac{r^{2} V(B)}{m(z) \frac{1}{4}}\right)^{1 /\left(p \kappa^{n}\right)} \inf _{Q_{\delta}} u^{2} .
$$


Since $n>\log (\delta r / 4) / \log 2$, we may estimate

$$
\left(\frac{r^{2} V(B)}{m(z) \frac{1}{4}}\right)^{1 / \kappa^{n}} \leq e^{(4 /(\delta r))^{c} \log \left(C r^{C}\right)} .
$$

Again $C$ is a constant which depends on $C_{1}$ and $C_{2}$. To obtain $C^{\prime} \delta^{-\gamma}$ as in (2.11), we must check

$$
\left(\frac{4}{\delta r}\right)^{c} \log \left(C r^{C}\right) \leq C^{\prime}-\gamma \log \delta
$$

for $\delta r \geq 2$. This may be done this way: either $\delta \leq r^{-1 / 2}$ and it suffices to note that $4 /(\delta r) \leq 4 / 2$ and use the term $-\gamma \log \delta$, either $\delta>r^{-1 / 2}$ and we use

$$
C^{\prime} \geq\left(\frac{4}{r^{1 / 2}}\right)^{c} \log \left(C r^{C}\right)
$$

The proof of (2.12) is identical, except that $u^{q}$ may be a supersolution, that's why we take $Q_{\delta}^{\prime \prime}$ instead of $Q_{\delta}$. We also use $u(t+\tau, z) \geq$ $e^{-\tau} u(t, z)$, that's another reason to cut $I$ by the highest values. In fact, having in mind the all-continuous result ([30, Corollary 3, p. 447]), we could keep $Q_{\delta}$. First, we should use a covering argument ([30, p. 448]) to avoid the use of Lemma 2.5 on $u^{q}$ for $q<1$. Then, instead of picking up the sup on the values $u(t+\tau, z)$, we could get it from the $u\left(t-\tau, z^{\prime}\right)$ where $z^{\prime} \sim z$. But this is only possible when $\delta r \geq 1$. Taking $Q_{\delta}^{\prime \prime}$ is somehow artificial but it has the great technical advantage that at this point of the proof, we have no more conditions like $\delta r \geq 1$ in Lemma 2.5 which compel us to treat separately cases when it is no longer possible to cut the space.

\subsection{About $\log u$, linking negative and positive exponents.}

Let us define the measure $\nu$ on $\mathbb{R} \times \Gamma$ as the product of Lebesgue measure and $V$. The next lemma states that the values of $\log u$ are glued to their (space) mean value at a time $\tau$ somehow like functions with BMO norm bounded, they cannot be much bigger on a large part before or much lower after. J. Moser's improvement in [23] is that this property and the (time and space) mean value inequalities are sufficient to link extrema to the (space) mean value of $\log u$ at a fixed time between $Q_{\ominus}$ and $Q_{\oplus}$, and thus to link extrema together. This last idea is the meaning of the abstract Lemma 2.9 . 
Lemma 2.8. Let $\bar{\eta}, \tau \in] 0,1\left[, B=B\left(x_{0}, r\right)\right.$ and $u$ any positive supersolution on $Q=\left[s, s+r^{2}\right] \times B$, there is a constant $m(u, \tau)$ such that for all $\lambda>0$,

and

$$
\nu\left(\left\{(t, z) \in K_{\oplus}: \log u(t, z)<m-\lambda\right\}\right) \leq \frac{C \nu(Q)}{\lambda}
$$

$$
\nu\left(\left\{(t, z) \in K_{\ominus}: \log u(t, z)>m+\lambda\right\}\right) \leq \frac{C \nu(Q)}{\lambda},
$$

where $K_{\oplus}=\left[s+\tau r^{2}, s+r^{2}\right] \times \bar{\eta} B, K_{\ominus}=\left[s, s+\tau r^{2}\right] \times \bar{\eta} B$ and $C$ depends only on $\bar{\eta}, \tau, C_{1}$ and $C_{2}$.

Proof. Let

$$
\psi(z)=1-\frac{d\left(x_{0}, z\right)}{[r]+1}
$$

( $[r]$ denotes the integer part of $r$ ) and $\bar{m}(x)=\sum_{y \notin B} \mu_{x y}$ so that for all $x \in B$,

$$
\begin{gathered}
m(x) \frac{\partial}{\partial t} u(t, x) \geq \sum_{y \in B} \mu_{x y}(u(t, y)-u(t, x))-\bar{m}(x) u(t, x) . \\
\frac{\partial}{\partial t} \sum_{x \in B} m(x) \psi^{2}(x)(-\log u(t, x)) \\
=\sum_{x \in B}-\psi^{2}(x) \frac{m(x) \frac{\partial}{\partial t} u(t, x)}{u(t, x)} \\
\leq \sum_{x, y \in B} \mu_{x y} \frac{-\psi^{2}(x)}{u(t, x)}(u(t, y)-u(t, x))+\sum_{x \in B} \psi^{2}(x) \bar{m}(x) \\
=\frac{1}{2} \sum_{x, y \in B} \mu_{x y}\left(\frac{\psi^{2}(y)}{u(t, y)}-\frac{\psi^{2}(x)}{u(t, x)}\right)(u(t, y)-u(t, x)) \\
+\sum_{x \in B} \psi^{2}(x) \bar{m}(x) .
\end{gathered}
$$

Now we show that

$$
\left(\frac{\psi^{2}(y)}{u(t, y)}-\frac{\psi^{2}(x)}{u(t, x)}\right)(u(t, y)-u(t, x))
$$

$$
\leq 36(\psi(y)-\psi(x))^{2}-\frac{1}{2} \min \left\{\psi^{2}(x), \psi^{2}(y)\right\} \frac{(u(t, y)-u(t, x))^{2}}{u(t, x) u(t, y)} .
$$


We may assume $u(t, x) \geq u(t, y)$ for that purpose.

Either

$$
\psi^{2}(x) \leq \frac{\psi^{2}(y)}{2}\left(1+\frac{u(t, x)}{u(t, y)}\right)
$$

then

$$
\begin{aligned}
\left(\frac{\psi^{2}(y)}{u(t, y)}-\frac{\psi^{2}(x)}{u(t, x)}\right)(u(t, y)-u(t, x)) \\
\\
\leq\left(\frac{\psi^{2}(y)}{u(t, y)}-\frac{\frac{\psi^{2}(y)}{2}\left(1+\frac{u(t, x)}{u(t, y)}\right)}{u(t, x)}\right)(u(t, y)-u(t, x)) \\
\quad=-\frac{1}{2} \psi^{2}(y) \frac{(u(t, y)-u(t, x))^{2}}{u(t, x) u(t, y)},
\end{aligned}
$$

and there is no need to use the other non-negative term $36(\psi(y)-$ $\psi(x))^{2}$.

$\mathrm{Or}$

$$
\psi^{2}(x) \geq \frac{\psi^{2}(y)}{2}\left(1+\frac{u(t, x)}{u(t, y)}\right) .
$$

First we estimate $u(t, y)-u(t, x)$ with $\psi(y)-\psi(x)$.

$$
\begin{aligned}
\frac{u(t, x)-u(t, y)}{u(t, y)} & =\frac{u(t, x)}{u(t, y)}-1 \\
& \leq \frac{2 \psi^{2}(x)}{\psi^{2}(y)}-2 \\
& =2 \frac{\psi(x)+\psi(y)}{\psi^{2}(y)}(\psi(x)-\psi(y)) .
\end{aligned}
$$

Thus,

$$
\begin{aligned}
\psi^{2}(y) \frac{(u(t, x)-u(t, y))^{2}}{u(t, x) u(t, y)} & \leq \psi^{2}(y) \frac{(u(t, x)-u(t, y))^{2}}{u^{2}(t, y)} \\
& \leq \psi^{2}(y)\left(2 \frac{\psi(x)+\psi(y)}{\psi^{2}(y)}(\psi(x)-\psi(y))\right)^{2} \\
& \leq 36(\psi(x)-\psi(y))^{2}
\end{aligned}
$$


Because the function $\psi$ is such that $\psi(y) \leq \psi(x) \leq 2 \psi(y)$ when $x \sim y$. We also obtain

$$
\begin{aligned}
\left(\frac{\psi^{2}(y)}{u(t, y)}-\frac{\psi^{2}(x)}{u(t, x)}\right) & (u(t, y)-u(t, x)) \\
& \leq\left(\frac{\psi^{2}(y)}{u(t, y)}-\frac{\psi^{2}(x)}{u(t, y)}\right)(u(t, y)-u(t, x)) \\
& =(\psi(x)+\psi(y)) \frac{u(t, x)-u(t, y)}{u(t, y)}(\psi(x)-\psi(y)) \\
& \leq 2 \frac{(\psi(x)+\psi(y))^{2}}{\psi^{2}(y)}(\psi(x)-\psi(y))^{2} \\
& \leq 18(\psi(x)-\psi(y))^{2} .
\end{aligned}
$$

Inequality (2.14) is proven because $18(\psi(x)-\psi(y))^{2}$ controls the two other terms.

We can now change (2.13) using the inequality (2.14),

$$
\begin{aligned}
& \frac{\partial}{\partial t} \sum_{x \in B} m(x) \psi^{2}(x)(-\log u(t, x)) \\
& +\frac{1}{2} \sum_{x, y \in B} \mu_{x y} \min \left\{\psi^{2}(x), \psi^{2}(y)\right\} \frac{(u(t, y)-u(t, x))^{2}}{u(t, x) u(t, y)} \\
& \quad \leq C \sum_{x, y \in B} \mu_{x y}(\psi(y)-\psi(x))^{2}+\sum_{x \in B} \psi^{2}(x) \bar{m}(x)
\end{aligned}
$$

Since

$$
(\log u(t, y)-\log u(t, x))^{2} \leq \frac{(u(t, y)-u(t, x))^{2}}{u(t, x) u(t, y)}
$$

(just check $(\log a)^{2} \leq(a-1)^{2} / a$ by differentiating two times), $x \sim y$ implies $|\psi(y)-\psi(x)| \leq 1 / r$ and $\bar{m}(x) \neq 0$ implies $|\psi(x)| \leq 1 / r$, this yields

$$
\begin{array}{r}
\frac{\partial}{\partial t} \sum_{x \in B} m(x) \psi^{2}(x)(-\log u(t, x)) \\
+\frac{1}{2} \sum_{x, y \in B} \mu_{x y} \min \left\{\psi^{2}(x), \psi^{2}(y)\right\}(\log u(t, y)-\log u(t, x))^{2} \\
\leq C \frac{V(B)}{r^{2}}
\end{array}
$$


Now use the weighted Poincaré inequality of Proposition 2.2 to estimate

$$
\begin{aligned}
& \sum_{x \in B} m(x) \psi^{2}(x)(-\log u(t, x)-W(t))^{2} \\
& \quad \leq C r^{2} \sum_{x, y \in B} \mu_{x y} \min \left\{\psi^{2}(x), \psi^{2}(y)\right\}(\log u(t, y)-\log u(t, x))^{2}
\end{aligned}
$$

where

$$
W(t)=\frac{\sum_{x \in B} m(x) \psi^{2}(x)(-\log u(t, x))}{\sum_{x \in B} m(x) \psi^{2}(x)} .
$$

Use also

$$
\sum_{x \in B} m(x) \psi^{2}(x) \geq \sum_{x \in B / 2} m(x)\left(\frac{1}{2}\right)^{2} \geq C V\left(\frac{B}{2}\right) \geq C^{\prime} V(B)
$$

and $x \in \bar{\eta} B$ implies $\psi(x) \geq 1-\bar{\eta}$. This way, we obtain two constants $c$ and $C$ depending only on $\bar{\eta}, C_{1}$ and $C_{2}$ such that

$$
\frac{\partial}{\partial t} W(t)+\frac{c}{\nu(Q)} \sum_{x \in \bar{\eta} B} m(x)(-\log (u(t, x))-W(t)) \leq C r^{-2}
$$

Setting $m=-W\left(s+\tau r^{2}\right)$, this yields the result (for precisions, follow litterally the argument on [30, p. 452]).

Lemma 2.9. Let $U_{\sigma}$ for $0 \leq \sigma \leq \varsigma \leq 1 / 2$ be subsets of a space with a measure $\nu$ such that $\sigma \leq \sigma^{\prime}$ implies $U_{\sigma} \supset U_{\sigma^{\prime}}$ and $\nu\left(U_{0}\right) \leq C \nu\left(U_{\varsigma}\right), f$ a positive measurable function on $U_{0}$ which satisfies

$$
\sup _{U_{\sigma^{\prime}}} f^{2} \leq C\left(C\left(\sigma^{\prime}-\sigma\right)^{-\gamma}\right)^{1 / p} \mathcal{M}\left(f, p, U_{\sigma}\right)
$$

for all $0<\sigma<\sigma^{\prime} \leq \varsigma$ and $p>0$ and

$$
\nu(\{\log f>\lambda\}) \leq \frac{C}{\lambda} \nu\left(U_{0}\right),
$$

for all $\lambda>0$. Then

$$
\sup _{U_{\varsigma}} f \leq A
$$


where $A$ depends only on $\varsigma, \gamma$ and $C$.

Proof. Set $\psi(\sigma)=\log \left(\sup _{U_{\sigma}} f^{2}\right)$. Dividing $U_{\sigma}$ into two sets $\log \left(f^{2}\right)$ $\leq \psi(\sigma) / 2$ and $\log \left(f^{2}\right) \geq \psi(\sigma) / 2$ yields

$$
\begin{aligned}
\mathcal{M}\left(f, p, U_{\sigma}\right)^{p} & \leq\left(e^{\psi(\sigma) / 2}\right)^{p}+\frac{1}{\nu\left(U_{\sigma}\right)} \frac{C}{\psi(\sigma) / 4} \nu\left(U_{0}\right) \sup _{U_{\sigma}} f^{2 p} \\
& \leq e^{p \psi(\sigma) / 2}+\frac{4 C^{2}}{\psi(\sigma)} e^{p \psi(\sigma)} \\
& \leq 2 e^{p \psi(\sigma) / 2}
\end{aligned}
$$

if we choose

$$
p=\frac{2}{\psi(\sigma)} \log \frac{\psi(\sigma)}{4 C^{2}}
$$

so that the two terms are equal. Then we apply (2.15),

$$
\begin{aligned}
\psi\left(\sigma^{\prime}\right) & \leq \log C+\frac{1}{p} \log \left(2 C\left(\sigma^{\prime}-\sigma\right)^{-\gamma} e^{\psi(\sigma) / 2}\right) \\
& \leq \log C+\frac{\psi(\sigma)}{2}\left(\frac{\log \left(2 C\left(\sigma^{\prime}-\sigma\right)^{-\gamma}\right)}{\log \left(\psi(\sigma) /\left(4 C^{2}\right)\right)}+1\right) .
\end{aligned}
$$

If

$$
\frac{\psi(\sigma)}{4 C^{2}} \geq\left(2 C\left(\sigma^{\prime}-\sigma\right)^{-\gamma}\right)^{2}
$$

and

$$
\log C \leq \frac{\psi(\sigma)}{8}
$$

then

$$
\psi\left(\sigma^{\prime}\right) \leq \frac{7}{8} \psi(\sigma)
$$

Thus, we always have

$$
\psi\left(\sigma^{\prime}\right) \leq \frac{7}{8} \psi(\sigma)+C^{\prime}\left(\sigma^{\prime}-\sigma\right)^{-2 \gamma}
$$

Take a positive decreasing sequence $\varsigma=\sigma_{0}>\cdots>\sigma_{i}>\sigma_{i+1}>\cdots$,

$$
\psi(\varsigma) \leq C^{\prime} \sum_{i=0}^{+\infty}\left(\frac{7}{8}\right)^{i}\left(\sigma_{i+1}-\sigma_{i}\right)^{-2 \gamma} \leq \mathrm{constant} \quad\left(=\log \left(A^{2}\right)\right)
$$


if we set $\sigma_{i}=\varsigma /(1+i)$.

\subsection{Proof of Theorem 2.1.}

Recall the notations of Definition 1.5 and Lemma 2.8, we set $\eta=$ $1 / 2, \bar{\eta}=3 / 4, \theta_{1}=1 / 6, \theta_{2}=1 / 3, \tau=1 / 2, \theta_{3}=3 / 4$ and $\theta_{4}=1$. Lemma 2.8 gives a reference value $m$ so that one can apply Lemma 2.9 to $f=e^{-m} u$ on $U_{0}=\left[s, s+\tau r^{2}\right] \times \bar{\eta} B$ with $U_{\sigma}=\left(U_{0}\right)_{\sigma}^{\prime \prime}$ for $\sigma \leq \varsigma=\bar{\eta}-\eta=1 / 4$. This way, $Q_{\ominus} \subset U_{\varsigma}$ and (2.15) is satisfied because of Lemma 2.7 and (2.8). This yields $\sup _{Q_{\ominus}}\left(e^{-m} u\right) \leq A$. Applying again Lemma 2.9 to $f=e^{m} u^{-1}$ on $U_{0}=\left[s+\tau r^{2}, s+\theta_{4} r^{2}\right] \times \bar{\eta} B$ with $U_{\sigma}=\left(U_{0}\right)_{\sigma}$ yields $\sup _{Q_{\oplus}}\left(e^{m} u^{-1}\right) \leq A$ and the Harnack inequality.

\section{Kernel estimates, discrete-time Harnack inequality and necessity of Poincaré inequality.}

\subsection{Continuous-time estimates.}

First, we give on-diagonal estimates. The regularity coming from the Harnack inequality shows that if one starts at $x$, one diffuses after a time $t$ on the ball $B(x, \sqrt{t})$. This is well known since the papers of D. G. Aronson [1] or of P. Li and S. T. Yau [19].

Proposition 3.1 (On-diagonal estimates). Assume $(\Gamma, \mu)$ satisfies $\mathcal{H}\left(C_{\mathcal{H}}\right)$, then

$$
\begin{gathered}
\mathcal{P}_{t}(x, y) \leq \frac{C_{\mathcal{H}} m(y)}{V(x, \sqrt{t})}, \quad \text { for all } x, y, t, \\
d(x, y)^{2} \leq t \text { implies } \mathcal{P}_{t}(x, y) \geq \frac{C_{\mathcal{H}}^{-2} m(y)}{V(x, \sqrt{t})}
\end{gathered}
$$

Proof. Applying the Harnack inequality to $\mathcal{P} .(\cdot, y)$ yields $\mathcal{P}_{t}(x, y) \leq$ $C_{\mathcal{H}} \mathcal{P}_{2 t}(z, y)$ for $z \in B(x, \sqrt{t})$. Thus,

$$
\mathcal{P}_{t}(x, y) \leq \frac{C_{\mathcal{H}}}{V(x, \sqrt{t})} \sum_{z \in B(x, \sqrt{t})} m(z) \mathcal{P}_{2 t}(z, y)
$$




$$
\begin{aligned}
& =\frac{C_{\mathcal{H}} m(y)}{V(x, \sqrt{t})} \sum_{z \in B(x, \sqrt{t})} \mathcal{P}_{2 t}(y, z) \\
& \leq \frac{C_{\mathcal{H}} m(y)}{V(x, \sqrt{t})}
\end{aligned}
$$

For the lower bound, we will use similarly $\mathcal{P}_{t / 2}(z, y) \leq C_{\mathcal{H}} \mathcal{P}_{t}(x, y)$ for $z \in B(x, \sqrt{t})$. But first, we define a function $u(\xi, \tau)$ solution of the parabolic equation in $[0, t] \times B(x, \sqrt{t})$ this way

$$
\begin{gathered}
u(\xi, \tau)=1, \quad \text { for all } \tau \in\left[0, \frac{t}{2}\right] \\
u(\xi, \tau)=\sum_{z \in B(x, \sqrt{t})} \mathcal{P}_{\tau-t / 2}(\xi, z), \quad \text { for all } \tau \in\left[\frac{t}{2}, t\right] .
\end{gathered}
$$

Applied to $u$ the Harnack inequality yields

$$
\begin{aligned}
C_{\mathcal{H}}^{-1}=C_{\mathcal{H}}^{-1} u\left(\frac{t}{2}, x\right) & \leq u(t, y) \\
& =\sum_{z \in B(x, \sqrt{t})} \mathcal{P}_{t / 2}(y, z) \\
& =\sum_{z \in B(x, \sqrt{t})} \frac{m(z)}{m(y)} \mathcal{P}_{t / 2}(z, y) \\
& \leq \sum_{z \in B(x, \sqrt{t})} \frac{C_{\mathcal{H}} m(z)}{m(y)} \mathcal{P}_{t}(x, y) \\
& =\frac{C_{\mathcal{H}} V(x, \sqrt{t})}{m(y)} \mathcal{P}_{t}(x, y) .
\end{aligned}
$$

These on-diagonal estimates yield the volume regularity.

Proposition 3.2. Assume $(\Gamma, \mu)$ satisfies $\mathcal{H}\left(C_{\mathcal{H}}\right)$. Then $D V\left(C_{\mathcal{H}}^{4}\right)$ is true.

ProOF.

$$
\frac{C_{\mathcal{H}}^{-2} m(x)}{V(x, r)} \leq \mathcal{P}_{r^{2}}(x, x) \leq C_{\mathcal{H}} \mathcal{P}_{4 r^{2}}(x, x) \leq C_{\mathcal{H}} \frac{C_{\mathcal{H}} m(x)}{V(x, 2 r)}
$$


Now we prove an off-diagonal upper bound which is more precise for $x$ and $y$ far apart. We still use the parabolic Harnack inequality as in Lemma 3.1 to estimate one term by a mean value and a second tool is the integrated maximum principle (see [13]).

Theorem 3.3 (Integrated maximum principle). If $u$ is a solution on $I \times \Gamma$ and $K(t, x)$ a positive and decreasing in $t$ function such that for all $t \in I$ and $x \sim y$,

$$
\begin{aligned}
(K(t, x)+ & K(t, y))^{2} \\
& \leq\left(\frac{\partial K}{\partial t}(t, x)-2 K(t, x)\right)\left(\frac{\partial K}{\partial t}(t, y)-2 K(t, y)\right),
\end{aligned}
$$

then the quantity

$$
I(t)=\sum_{x \in \Gamma} m(x) u^{2}(t, x) K(t, x)
$$

is decreasing in $t \in I$.

ProOF.

$$
\begin{aligned}
I^{\prime}(t)= & \sum_{x \in \Gamma} m(x) u^{2}(t, x) \frac{\partial K}{\partial t}(t, x) \\
& +\sum_{x, y \in \Gamma} 2 \mu_{x y}(u(t, y)-u(t, x)) u(t, x) K(t, x) .
\end{aligned}
$$

Since the weights $\mu_{x y}$ are symmetric,

$$
\begin{aligned}
\sum_{x \in \Gamma} m(x) u^{2}(t, x) & \frac{\partial K}{\partial t}(t, x) \\
= & \sum_{x, y \in \Gamma} \frac{\mu_{x y}}{2}\left(u^{2}(t, x) \frac{\partial K}{\partial t}(t, x)+u^{2}(t, y) \frac{\partial K}{\partial t}(t, y)\right)
\end{aligned}
$$

and

$$
\begin{aligned}
& \sum_{x, y \in \Gamma} 2 \mu_{x y}(u(t, y)-u(t, x)) u(t, x) K(t, x) \\
& \quad=\sum_{x, y \in \Gamma} \mu_{x y}(u(t, y)-u(t, x))(u(t, x) K(t, x)-u(t, y) K(t, y))
\end{aligned}
$$


This yields

$$
\begin{aligned}
I^{\prime}(t)=\sum_{x, y \in \Gamma} \mu_{x y} & \left(\frac{u^{2}(t, x)}{2}\left(\frac{\partial K}{\partial t}(t, x)-2 K(t, x)\right)\right. \\
& +u(t, x) u(t, y)(K(t, x)+K(t, y)) \\
& \left.+\frac{u^{2}(t, y)}{2}\left(\frac{\partial K}{\partial t}(t, y)-2 K(t, y)\right)\right)
\end{aligned}
$$

$\leq 0$,

because of (3.16).

Construction of a function $K$. Take $\xi(t, x)=\log (K(t, x)),(3.16)$ becomes

$(3.17) \chi(\xi(t, x)-\xi(t, y))+\frac{\partial \xi}{\partial t}(t, x)+\frac{\partial \xi}{\partial t}(t, y) \leq \frac{1}{2} \frac{\partial \xi}{\partial t}(t, x) \frac{\partial \xi}{\partial t}(t, y)$

where $\chi(s)=\cosh (s)-1$. Note that $\chi(s) \sim s^{2} / 2$ for $s$ small so that (3.17) may be connected to the following eikonal inequation for the heat equation on a continuous geometry

$$
\frac{\partial \xi}{\partial t}+\frac{1}{2}|\nabla \xi|^{2} \leq 0
$$

with a solution $K=e^{\xi}=e^{d^{2} / t}$ where $d$ is a distance function of $x$. Our parabolic equation should have been normalized to obtain the same coefficients. This difference and differential inequation (3.17) contains only first-order terms, that's why we get nice solutions considering its Legendre associate. For instance,

$$
\xi(t, x)=\zeta(t, d(x))=\max _{\lambda}\{\lambda d(x)-\chi(\lambda) t\}
$$

is a solution if $x \sim y$ implies $|d(x)-d(y)| \leq 1$. Indeed, note $\lambda(t, x)$ a value for which the maximum is reached,

$$
\frac{\partial \xi}{\partial t}(t, x)=-\chi(\lambda(t, x))
$$

and

$$
|\xi(t, x)-\xi(t, y)| \leq \max \{\lambda(t, x), \lambda(t, y)\}
$$


We obtain

$$
\begin{gathered}
\lambda(t, x)=\arg \sinh \left(\frac{d(x)}{t}\right) \\
\zeta(t, d)=d \arg \sinh \left(\frac{d}{t}\right)-t\left(\sqrt{1+\frac{d^{2}}{t^{2}}}-1\right) .
\end{gathered}
$$

It will be useful to note that, since $\partial \zeta / \partial d=\lambda$,

$$
\left\{\begin{array}{l}
\zeta(t, d) \leq \frac{1}{2} \frac{d^{2}}{t}, \\
d \leq C t \text { implies } \zeta(d, t) \geq \frac{\arg \sinh C}{2 C} \frac{d^{2}}{t} .
\end{array}\right.
$$

Denote $E[t, d]=e^{\zeta(t, d)}$ in the sequel. This function has already been introduced by E. B. Davies in [8] with his semigroup perturbation argument (see [7]). With this argument and Harnack inequality, L. SaloffCoste proves Gaussian upper bounds in [28] using ideas of [38], [39]. We adapt this proof to the use of the integrated maximum principle in the next proposition.

Proposition 3.4 (Off-diagonal upper bound). Assume ( $\Gamma, \mu)$ satisfies $\mathcal{H}\left(C_{\mathcal{H}}\right)$, then for all $x, y, t$,

$$
\mathcal{P}_{t}(x, y) \leq \frac{C m(y)}{\sqrt{V(x, \sqrt{t}) V(y, \sqrt{t}) E[6 t, d(x, y)}]}
$$

where $C$ depends only on $C_{\mathcal{H}}$.

Proof. Consider the following solution of the parabolic equation,

$$
u(\tau, \xi)=\sum_{\eta \in B(y, \sqrt{t})} p_{t}(\eta, x) p_{\tau}(\xi, \eta)
$$

This will be useful to estimate $A(t)=\sum_{\eta \in B(y, \sqrt{t})} m(\eta) p_{t}^{2}(\eta, x)$. Indeed we apply Theorem 3.3 between 0 and $2 t$ to

$$
I(\tau)=\sum_{\xi \in \Gamma} m(\xi) u^{2}(\tau, \xi) E[t+\tau, d(y, \xi)]
$$


Since $u(0, \xi)=0$ if $\xi \notin B(y, \sqrt{t})$ and $u(0, \xi)=p_{t}(\xi, x)$ if $\xi \in B(y, \sqrt{t})$, we have

$$
I(0)=\sum_{\xi \in B(y, \sqrt{t})} m(\xi) p_{t}^{2}(\xi, x) E[t, d(y, \xi)] \leq C A(t)
$$

Just note that $d \leq \sqrt{t}$ implies $E[t, d] \leq e^{1 / 2}$, see (3.18).

In order to give a lower bound for $I(2 t)$, we use

$$
p_{2 t}(\xi, \eta) \geq C_{\mathcal{H}}^{-1} p_{t}(x, \eta)
$$

so that

$$
u(2 t, \xi) \geq \frac{C_{\mathcal{H}}^{-1}}{m(x)} A(t)
$$

for $\xi \in B(x, \sqrt{t})$. This yields

$$
\begin{aligned}
I(2 t) & =\sum_{\xi \in \Gamma} m(\xi) u^{2}(2 t, \xi) E[3 t, d(y, \xi)] \\
& \geq C_{\mathcal{H}}^{-2} \sum_{\xi \in B(x, \sqrt{t})} \frac{m(\xi)}{m(x)^{2}} A(t)^{2} E[3 t, d(y, B(x, \sqrt{t}))] .
\end{aligned}
$$

Since $I(2 t) \leq I(0)$, we get

$$
A(t) \leq \frac{C m(x)^{2}}{V(x, \sqrt{t}) E[3 t, d(y, B(x, \sqrt{t}))]} .
$$

Again the Harnack inequality gives

$$
\begin{aligned}
p_{t}^{2}(x, y) & =\frac{m(y)^{2}}{m(x)^{2}} p_{t}^{2}(y, x) \\
& \leq \frac{C_{\mathcal{H}}^{2} m(y)^{2}}{m(x)^{2} V(y, \sqrt{t})} \sum_{\eta \in B(y, \sqrt{t})} m(\eta) p_{2 t}^{2}(\eta, x) \\
& =\frac{C_{\mathcal{H}}^{2} m(y)^{2}}{m(x)^{2} V(y, \sqrt{t})} A(2 t) \\
& \leq \frac{C m(y)^{2}}{V(x, \sqrt{2 t}) V(y, \sqrt{t}) E[6 t, d(y, B(x, \sqrt{2 t}))]}
\end{aligned}
$$


The proposition follows because of the volume regularity and

$$
E[6 t, d(y, B(x, \sqrt{2 t}))] \geq c E[6 t, d(x, y)] .
$$

REMARK 1. Instead of $E[6 t, d(x, y)]$, we could get $E[\lambda t, d(x, y)]$ for any $\lambda>1$, the constant $C$ depending also on $\lambda$. We would just have to apply the Harnack inequality between $t$ and $(1+\varepsilon) t$. Furthermore, we could have been more precise for the choice of a function $E$ (using both $\chi(\lambda(x))$ and $\chi(\lambda(y))$ instead of only the biggest). All of these manipulations tend to obtain the analog of $e^{d^{2} /((4+\varepsilon) t)}$ for $d / t$ small. Don't forget the normalization of the parabolic equation to compare.

REMARK 2. One might find the function $E$ too complicated, but [25] explained that it is not purely technical. To understand it, one can take it as $e^{c d^{2} / t}$ for $d / t$ small and $(d / t)^{d} e^{-d}$ for $d / t$ huge glued together. The second value is adapted to the fact that when $t \longrightarrow 0$,

$$
\mathcal{P}_{t}(x, y) \sim \frac{p_{d(x, y)}(x, y) t^{d(x, y)}}{d(x, y) !}
$$

so at least in this case the function $E$ gives an optimal upper bound.

\subsection{Discrete-time estimates.}

Assume $\Delta(\alpha)$ is true so that we can consider the positive submarkovian kernel $\bar{p}=p-\alpha \delta$ (this means $\bar{p}(x, y)=p(x, y)-\alpha \delta(x, y)$, then $\bar{p}_{n}(x, y)$ is defined as in (1.1)). Now compute $\mathcal{P}_{n}$ and $p_{n}$ with $\bar{p}$

$$
\begin{gathered}
\mathcal{P}_{n}(x, y)=e^{(\alpha-1) n} \sum_{k=0}^{+\infty} \frac{n^{k}}{k !} \bar{p}_{k}(x, y)=\sum_{k=0}^{+\infty} a_{k} \bar{p}_{k}(x, y) \\
p_{n}(x, y)=\sum_{k=0}^{n} C_{n}^{k} \alpha^{n-k} \bar{p}_{k}(x, y)=\sum_{k=0}^{n} b_{k} \bar{p}_{k}(x, y)
\end{gathered}
$$

To compare the two sums we study $c_{k}=b_{k} / a_{k}$ for $0 \leq k \leq n$,

$$
c_{k}=\frac{n ! \alpha^{n-k}}{(n-k) ! e^{(\alpha-1) n} n^{k}} .
$$




\section{Lemma 3.5.}

$$
\begin{gathered}
0 \leq k \leq n \text { implies } c_{k} \leq C(\alpha), \\
n \geq \frac{a^{2}}{\alpha^{2}} \text { and }|k-(1-\alpha) n| \leq a \sqrt{n} \text { imply } c_{k} \geq C(a, \alpha)>0 .
\end{gathered}
$$

The condition $n \geq a^{2} / \alpha^{2}$ ensures that $a \sqrt{n} \leq \alpha n$. We shall consider only $\alpha \leq 1 / 4$ so that we always have $n / 2 \leq k \leq n$ in the second assertion.

Proof. The $c_{k}$ 's follow the recurrence formula $c_{k+1}=c_{k}(n-k) /(\alpha n)$, so they reach a maximum for $\mathrm{k}$ around the real $(1-\alpha) n$. Let us use the Gamma function, $\Gamma(n+1)=n$ ! and $t^{t} e^{-t} \sqrt{t} / C \leq \Gamma(t+1) \leq C t^{t} e^{-t} \sqrt{t}$. Set $c_{t}=\Gamma(n+1) \alpha^{n-t} / \Gamma(n-k+1) e^{(\alpha-1) t} n^{t}$. Similarly, it reaches its maximum for $t=(1-\alpha) n$. Thus,

$$
\begin{aligned}
c_{k} & \leq \frac{\Gamma(n+1) \alpha^{\alpha n}}{\Gamma(\alpha n+1) e^{(\alpha-1) n} n^{(1-\alpha) n}} \\
& =\frac{\Gamma(n+1)}{n^{n} e^{-n}} \frac{(\alpha n)^{\alpha n} e^{-\alpha n}}{\Gamma(\alpha n+1)} \\
& \leq C \sqrt{n} \frac{C}{\sqrt{\alpha n}} \\
& =\frac{C^{2}}{\sqrt{\alpha}} .
\end{aligned}
$$

Next, we prove the second assertion of the lemma. Again, because of $c_{k}$ 's variations, we only check this for $k \approx(1-\alpha) n \pm a \sqrt{n}$. For instance, for $(1-\alpha) n-a \sqrt{n} \leq k \leq(1-\alpha) n$,

$$
\begin{aligned}
c_{k} & =\frac{n ! \alpha^{n-k}}{(n-k) ! e^{(\alpha-1) n} n^{k}} \\
& \geq \frac{\Gamma(n+1) \alpha^{\alpha n+a \sqrt{n}}}{\Gamma(\alpha n+a \sqrt{n}+1) e^{(\alpha-1) n} n^{(1-\alpha) n-a \sqrt{n}}} \\
& \geq \frac{1}{C^{2}} \frac{e^{a \sqrt{n}}}{\left(1+\frac{a \sqrt{n}}{\alpha n}\right)^{\alpha n+a \sqrt{n}}} \frac{\sqrt{n}}{\sqrt{\alpha n+a \sqrt{n}}} \\
& \geq \frac{1}{C^{2}} e^{a \sqrt{n}-(\alpha n+a \sqrt{n}) \log (1+a /(\alpha \sqrt{n}))} 1
\end{aligned}
$$




$$
\begin{aligned}
& \geq \frac{1}{C^{2}} e^{a \sqrt{n}-(\alpha n+a \sqrt{n}) a /(\alpha \sqrt{n})} \\
& =\frac{1}{C^{2}} e^{-a^{2} / \alpha}
\end{aligned}
$$

This technical result is the key to compare $p$ and $\mathcal{P}$. One side is easy now.

Theorem 3.6. Assume $(\Gamma, \mu)$ satisfies $p(x, x) \geq \alpha>0$ for all $x$ in $\Gamma$. Then, for all $x, y, n$,

$$
p_{n}(x, y) \leq C(\alpha) \mathcal{P}_{n}(x, y) .
$$

It is the case when $\Delta(\alpha)$ is true. This theorem may be applied in many other situations (with any volume growth) when it is easier to work on $\mathcal{P}$. When no hypothesis is assumed on $p(x, x)$, see the comment after Definition 1.3 about $\left(\Gamma, \mu^{(2)}\right)$. For instance, on a locally uniformly finite (by $N$ ) non-weighted graph $\left(\mu_{x y} \in\{0,1\}\right), p_{2}(x, x) \geq 1 / N$.

The other side of the comparison is more intricate.

Proposition 3.7 (On-diagonal estimates). Assume $(\Gamma, \mu)$ satisfies $D V\left(C_{1}\right), P\left(C_{2}\right)$ and $\Delta(\beta)$. Then, there exist $c_{d}, C_{d}>0$, depending only on $C_{1}, C_{2}$ and $\beta$, such that

$$
\begin{gathered}
p_{n}(x, y) \leq \frac{C_{d} m(y)}{V(x, \sqrt{n})}, \quad \text { for all } x, y, n \\
d(x, y)^{2} \leq n \text { implies } p_{n}(x, y) \geq \frac{c_{d} m(y)}{V(x, \sqrt{n})}
\end{gathered}
$$

Proof. The first assertion follows from Theorem 3.6 and the upper bound in Proposition 3.1. To deduce the second assertion from the lower bound in Proposition 3.1, we will have to prove that in the sum (3.19), the terms for $|k-(1-\alpha) n| \leq a \sqrt{n}$ contain half of the whole sum. First we will set $\alpha=\beta / 2$ (this will be useful later when we apply the upper bound to a Markov kernel $p^{\prime}$ ). Now, to prove that the lower bound for $\mathcal{P}$ implies one for $p$, it will be sufficient to prove that for all $\varepsilon>0$, there exists $a$,

$$
\sum_{|k-(1-\alpha) n|>a \sqrt{n}} a_{k} \bar{p}_{k}(x, y) \leq \frac{\varepsilon m(y)}{V(x, \sqrt{n})} .
$$


We will take $\varepsilon=C_{\mathcal{H}}^{-2} / 2$, the desired lower bound will be proved for $n \geq N=a^{2} / \alpha^{2}$. For $n \leq N$, the condition $\Delta(\beta)$ gives $p_{n}(x, y) \geq \beta^{N}$.

We can apply the upper bound to the Markov kernel $p^{\prime}=\bar{p} /(1-\alpha)$. Indeed, it is generated by weights $\mu_{x y}^{\prime}$

$$
\begin{gathered}
\mu_{x x}^{\prime}=\frac{\mu_{x x}-\alpha m(x)}{1-\alpha} \geq \alpha m(x), \\
\mu_{x y}^{\prime}=\frac{\mu_{x y}}{1-\alpha}, \quad \text { if } x \neq y, \\
m^{\prime}(x)=m(x) .
\end{gathered}
$$

Thus, the volume is identical and $P\left(C_{2}\right)$ is still satisfied because weights $\mu_{x y}$ for $x \neq y$ have increased. This yields $p_{k}^{\prime}(x, y) \leq C_{d}^{\prime} m(y) / V(x, \sqrt{k})$, hence $\bar{p}_{k}(x, y) \leq C_{d}^{\prime} m(y)(1-\alpha)^{k} / V(x, \sqrt{k})$. Next, we have to get the estimate

$$
\sum_{|k-(1-\alpha) n|>a \sqrt{n}} e^{(\alpha-1) n} \frac{((1-\alpha) n)^{k}}{k !} \frac{1}{V(x, \sqrt{k})} \leq \frac{\varepsilon^{\prime}}{V(x, \sqrt{n})} .
$$

The sum for $k>(1-\alpha) n+a \sqrt{n}$ is easier because we simply use $V(x, \sqrt{k}) \geq V(x, \sqrt{n / 2}) \geq V(x, \sqrt{n} / 2) \geq V(x, \sqrt{n}) / C_{1}$. Then, we obtain the $k+1^{\text {th }}$ term of the sum if we multiply the $k^{\text {th }}$ term by $(1-\alpha) n /(k+1)$. So we estimate this part by a geometric sum,

$$
\begin{aligned}
& \sum_{k>(1-\alpha) n+a \sqrt{n}} e^{(\alpha-1) n} \frac{((1-\alpha) n)^{k}}{k !} \frac{1}{V(x, \sqrt{k})} \\
\leq & e^{(\alpha-1) n} \frac{((1-\alpha) n)^{(1-\alpha) n+a \sqrt{n}}}{\Gamma((1-\alpha) n+a \sqrt{n}+1)} \frac{C_{1}}{V(x, \sqrt{n})} \frac{1}{1-\frac{(1-\alpha) n}{(1-\alpha) n+a \sqrt{n}}} \\
\leq & C C_{1} e^{a \sqrt{n}-((1-\alpha) n+a \sqrt{n}) \log (1+a /((1-\alpha) \sqrt{n}))} \\
& \frac{1}{V(x, \sqrt{n})} \underbrace{\frac{1}{\sqrt{(1-\alpha) n+a \sqrt{n}}} \frac{(1-\alpha) n+a \sqrt{n}}{\underbrace{\prime}}}_{\leq \frac{1}{a} \text { because } n \geq \frac{a^{2}}{\alpha^{2}}} \\
\leq & \frac{\varepsilon^{\prime} / 2}{V(x, \sqrt{n})},
\end{aligned}
$$


with a good choice of $a$. Indeed, since $\log (1+u) \geq u /(u+1)$, the argument of the exponential function appears to be negative.

To deal with $k<(1-\alpha) n-a \sqrt{n}$, we must be careful with the factors $V(x, \sqrt{k}) / V(x, \sqrt{k-1})$ when we compute the $k-1^{\text {th }}$ term from the $k^{\text {th }}$. A rough application of the volume regularity gives $V(x, \sqrt{k}) \leq$ $C_{1} V(x, \sqrt{k-1})$. So for the terms $k \leq(1-\alpha) n /\left(2 C_{1}\right)$, the $k-$ $1^{\text {th }}$ term is less than one half of the $k^{\text {th }}$ term and the estimation is straightforward. Now for the other terms we bound all $1 / V(x, \sqrt{k})$ by $1 / V\left(x, \sqrt{(1-\alpha) n /\left(2 C_{1}\right)}\right)$, then the same computation as for $k>$ $(1-\alpha) n+a \sqrt{n}$ shows the estimate with $1 / V\left(x, \sqrt{(1-\alpha) n /\left(2 C_{1}\right)}\right)$ which is less than $C / V(x, \sqrt{n})$ if we apply many times the volume regularity.

Now we prove off-diagonal upper and lower bounds.

Theorem 3.8 (Off-diagonal estimates). Assume $(\Gamma, \mu)$ satisfies $D V\left(C_{1}\right), P\left(C_{2}\right)$ and $\Delta(\alpha)$. Then, there exist positive $c_{l}, C_{l}, C_{r}$ and $c_{r}$ depending only on $C_{1}, C_{2}$ and $\alpha$ such that $G\left(c_{l}, C_{l}, C_{r}, c_{r}\right)$ is true.

Proof OF THE UPPER BOUND. It is a consequence of Theorem 3.6 and Proposition 3.4.

$$
\begin{aligned}
p_{n}(x, y) & \leq \frac{C m(y)}{\sqrt{V(x, \sqrt{n}) V(y, \sqrt{n}) E[6 n, d(x, y)]}} \\
& \leq \frac{C m(y)}{\sqrt{V(x, \sqrt{n}) V(y, \sqrt{n})}} e^{-c d(x, y)^{2} / n}
\end{aligned}
$$

for $d(x, y) \leq n$ because of (3.18).

Now use

$$
\begin{aligned}
V(x, \sqrt{n}) \leq & V(y, d(x, y)+\sqrt{n}) \\
\leq & C_{1}\left(\frac{d(x, y)+\sqrt{n}}{\sqrt{n}}\right)^{\log C_{1} / \log 2} V(y, \sqrt{n}), \\
p_{n}(x, y) \leq & \frac{C \sqrt{C_{1}} m(y)}{V(x, \sqrt{n})}\left(\frac{d(x, y)+\sqrt{n}}{\sqrt{n}}\right)^{\log C_{1} / 2 \log 2} \\
& \cdot e^{-(c / 2) d(x, y)^{2} / n} e^{-(c / 2) d(x, y)^{2} / n} .
\end{aligned}
$$

It is clear that the factor

$$
\left(\frac{d(x, y)+\sqrt{n}}{\sqrt{n}}\right)^{\log C_{1} / 2 \log 2} e^{-(c / 2) d(x, y)^{2} / n}
$$


is bounded.

ProOF OF THE LOWER BOUnd. It is well-known that the Gaussian lower bound follows from the on-diagonal one. So let us apply many times the second assertion of Proposition 3.7. We set $n=n_{1}+\cdots+n_{j}$, $x=x_{0}, x_{1}, \ldots, x_{j}=y$ and $B_{0}=\{x\}, B_{i}=B\left(x_{i}, r_{i}\right), B_{j}=\{y\}$ such that

$$
\left\{\begin{array}{l}
j-1 \leq C \frac{d(x, y)^{2}}{n}, \\
r_{i} \geq c \sqrt{n_{i+1}}, \quad \text { so that } z \in B_{i} \text { imply } V\left(z, \sqrt{n_{i+1}}\right) \leq A V\left(B_{i}\right) \\
\sup _{\substack{z \in B_{i-1} \\
z^{\prime} \in B_{i}}} d\left(z, z^{\prime}\right)^{2} \leq n_{i}, \text { so that } p_{n_{i}}\left(z, z^{\prime}\right) \geq \frac{c_{d} m\left(z^{\prime}\right)}{V\left(z, \sqrt{n_{i}}\right)} .
\end{array}\right.
$$

We will see below how to construct this decomposition. It is a purely technical problem (cutting in a discrete context).

It will be sufficient to prove the Gaussian lower bound since

$$
\begin{aligned}
& p_{n}(x, y) \\
& \geq \sum_{\left(z_{1}, \ldots, z_{j-1}\right) \in B_{1} \times \cdots \times B_{j-1}} p_{n_{1}}\left(x, z_{1}\right) p_{n_{2}}\left(z_{1}, z_{2}\right) \cdots p_{n_{j}}\left(z_{j-1}, y\right) \\
& \geq \sum_{\left(z_{1}, \ldots, z_{j-1}\right) \in B_{1} \times \cdots \times B_{j-1}} \frac{c_{d} m\left(z_{1}\right)}{V\left(x, \sqrt{n_{1}}\right)} \frac{c_{d} m\left(z_{2}\right)}{V\left(z_{1}, \sqrt{n_{2}}\right)} \cdots \frac{c_{d} m(y)}{V\left(z_{j-1}, \sqrt{n_{j}}\right)} \\
& \geq c_{d}^{j} A^{1-j} \sum_{\left(z_{1}, \ldots, z_{j-1}\right) \in B_{1} \times \cdots \times B_{j-1}} \frac{m\left(z_{1}\right)}{V\left(x, \sqrt{n_{1}}\right)} \frac{m\left(z_{2}\right)}{V\left(B_{1}\right)} \cdots \frac{m(y)}{V\left(B_{j}\right)} \\
& =\frac{c_{d} m(y)}{V\left(x, \sqrt{n_{1}}\right)}\left(\frac{c_{d}}{A}\right)^{j-1} .
\end{aligned}
$$

We just have to choose $C_{l} \geq C \log \left(A / c_{d}\right)$.

Decomposition. Consider three cases.

If $d(x, y) \geq n / 100$, then we can set $j=n, n_{i} \equiv 1, B_{i}=\left\{x_{i}\right\}$ (for instance, $\left.r_{i} \equiv 1 / 2\right)$ and choose $d\left(x_{i}, x_{i+1}\right) \leq 1$.

If $d(x, y)^{2} \leq n$, then we can set $j=1$ (in fact Proposition 3.7 has not to be iterated). 
Otherwise, set

$$
j=\left[10 \frac{d(x, y)^{2}}{n}\right] \geq 10 .
$$

This way, $n / j$ and $d(x, y) / j$ are bigger than 10 and

$$
\left(\frac{d(x, y)}{j}\right)^{2} \leq \frac{n}{9 j}
$$

so we can choose $n_{i} \approx n / j(i . e .[n / j]$ or $[n / j]+1)$ and

$$
d\left(x_{i}, x_{i}+1\right) \leq r_{i} \approx \frac{d(x, y)}{j}
$$

\subsection{Discrete-time Harnack inequality.}

We will prove the discrete-time Harnack inequality thanks to the Gaussian estimates. The method is based on [11, Section 3]. Denote $B=B\left(x_{0}, R\right)$ where $R \in \mathbb{N}^{*}$, with boundary $\partial B=\left\{x: d\left(x_{0}, x\right)=R\right\}$. The idea of the proof is that for $(\nu, \xi) \in \partial Q$ and $(n, x) \in Q_{\ominus}$ or $Q_{\oplus}$, $p_{n-\nu}(x, \xi)$ 's lower and upper bounds differ only by a constant. The difficulty is that the solution $u$ on $Q$ is not a combination of $p_{n-\nu}(x, \xi)$ but of $U_{n-\nu}(x, \xi)$ where $U_{n}(x, y)$ is the solution for $(n, x) \in \mathbb{N} \times B$ satisfying $U_{0}(x, y)=\delta(x, y)$ and $U_{n}(x, y)=0$ for $n>0$ and $x \in \partial B$. Obviously $U_{n}(x, y) \leq p_{n}(x, y)$, so only the lower bound needs some work.

Lemma 3.9. Assume $(\Gamma, \mu)$ satisfies $G\left(c_{l}, C_{l}, C_{r}, c_{r}\right)$. Then, there exist $\varepsilon, c>0$ depending only on $c_{l}, C_{l}, C_{r}$ and $c_{r}$ such that

$$
U_{n}(x, y) \geq \frac{c m(y)}{V\left(x_{0}, 2 \varepsilon R\right)},
$$

whenever

$$
\left\{\begin{array}{l}
(\varepsilon R)^{2} \leq n \leq(2 \varepsilon R)^{2}, \\
x \in B\left(x_{0}, \varepsilon R\right) \\
y \in B\left(x_{0}, 2 \varepsilon R\right) \\
d(x, y) \leq n
\end{array}\right.
$$


Proof. The idea (see [11, Lemma 5.1]) is that if $d(x, \partial B)$ is big enough, $p-U$ is small and the lower bound for $p$ applies to $U$. First note that

$$
p_{n}(x, y) \geq \frac{2 c m(y)}{V\left(x_{0}, 2 \varepsilon R\right)},
$$

where $c=c_{l} e^{-9 C_{l}} / 2$. Now, write

$$
r(n, x)=p_{n}(x, y)-U_{n}(x, y)=\sum_{\substack{\xi \in \partial B \\ \nu \leq n}} a(\nu, \xi) p_{n-\nu}(x, \xi)
$$

where $a(\xi, \nu) \geq 0$. These coefficients may be constructed by recurrence on $\nu$. Another point of view is that $(m(\xi) / m(y)) a(\nu, \xi)$ is the probability to reach $\partial B$ for the first time at $\xi$ after $\nu$ steps. That's why

$$
\sum_{\nu, \xi} \frac{m(\xi)}{m(y)} a(\nu, \xi) \leq 1 .
$$

We can check it this way

$$
\begin{aligned}
1 & =\sum_{x} \frac{m(x)}{m(y)} p_{n}(x, y) \\
& \geq \sum_{x} \frac{m(x)}{m(y)} r(n, x) \\
& =\sum_{x, \nu, \xi} \frac{m(x)}{m(y)} a(\nu, \xi) p_{n-\nu}(x, \xi) \\
& =\sum_{\nu, \xi}(a(\nu, \xi) \underbrace{\sum_{x} \frac{m(x)}{m(y)} p_{n-\nu}(x, \xi)}_{=m(\xi) / m(y)}) .
\end{aligned}
$$

To estimate $r(n, x)$ we use the Gaussian upper bound.

$$
\begin{aligned}
\frac{m(y)}{m(\xi)} p_{n-\nu}(x, \xi) & \leq \frac{C_{r} m(y)}{V(x, \sqrt{n-\nu})} e^{-c_{r} d(x, y)^{2} /(n-\nu)} \\
& \leq\left(C_{r} \frac{V(x, 2 \varepsilon R)}{V(x, \sqrt{n-\nu})} e^{-c_{r}((1-\varepsilon) R)^{2} /(n-\nu)}\right) \frac{m(y)}{V(x, 2 \varepsilon R)} \\
& \leq \frac{c m(y)}{V\left(x_{0}, 2 \varepsilon R\right)}
\end{aligned}
$$


with a good choice of $\varepsilon$. The lemma follows.

Theorem 3.10. Assume $(\Gamma, \mu)$ satisfies $G\left(c_{l}, C_{l}, C_{r}, c_{r}\right)$, then there exists $C_{H}>0$ such that $H\left(C_{H}\right)$ is true.

ProOF. Let us first point out that the Gaussian lower bound yields a volume regularity. The following argument,

$$
\begin{aligned}
1 & \geq \sum_{y \in B(x, 2 r)} p_{r^{2}}(x, y) \\
& \geq \sum_{y \in B(x, 2 r)} \frac{c_{l} m(y)}{V(x, r)} e^{-C_{l}(2 r)^{2} / r^{2}} \\
& =c_{l} e^{-4 C_{l}} \frac{V(x, 2 r)}{V(x, r)}
\end{aligned}
$$

is correct for $r$ integer and $r \geq 2$ (because we need $d(x, y) \leq r^{2}$ ). This extends to other values thanks to $\Delta(\alpha)$ (which is an immediate consequence of $\left.G\left(c_{l}, C_{l}, C_{r}, c_{r}\right)\right)$.

Now we prove the Harnack inequality for $\eta=\varepsilon, \theta_{1}=\varepsilon^{2} / 2, \theta_{2}=\varepsilon^{2}$, $\theta_{3}=2 \varepsilon^{2}, \theta_{4}=4 \varepsilon^{2}$ and $r=R \in \mathbb{N}^{*}$ in the notations of Definition 1.6.

Let $u$ be a solution on $Q$, there is a decomposition

$$
v(n, x)=\sum_{\substack{\nu \leq n \\ \xi \in \partial B\left(x_{0}, 2 \varepsilon R\right) \\ \text { or } \\ \nu=0 \\ \xi \in B\left(x_{0}, 2 \varepsilon R\right)}} a(\nu, \xi) U_{n-\nu}(x, \xi),
$$

with non-negative $a(\nu, \xi)$ such that $u(n, x)=v(n, x)$ if $x \in B\left(x_{0}, 2 \varepsilon R\right)$. Again the coefficients may be constructed by recurrence on $\nu$, the key is to keep $v \leq u$ everywhere.

Thus, it will be sufficient to prove the Harnack inequality for the terms $U_{{ }_{-\nu}}(\cdot, \xi)$, this means $U_{n_{\ominus}-\nu}\left(x_{\ominus}, \xi\right) \leq C U_{n_{\oplus}-\nu}\left(x_{\oplus}, \xi\right)$ for $\left(n_{\ominus}, x_{\ominus}\right) \in Q_{\ominus},\left(n_{\oplus}, x_{\oplus}\right) \in Q_{\oplus}, \nu \leq \theta_{2} R^{2}$ and $d\left(x_{\ominus}, x_{\oplus}\right) \leq n_{\oplus}-n_{\ominus}$. The lower bound is a consequence of Lemma 3.9 if $d\left(x_{\oplus}, \xi\right) \leq n_{\oplus}-\nu$,

$$
U_{n_{\oplus}-\nu}\left(x_{\oplus}, \xi\right) \geq \frac{c m(\xi)}{V\left(x_{0}, 2 \varepsilon R\right)}
$$


for $x_{\oplus} \in B\left(x_{0}, \eta R\right)=B\left(x_{0}, \varepsilon R\right)$ and $\theta_{3} R^{2} \leq n_{\oplus} \leq \theta_{4} R^{2}$. If $d\left(x_{\oplus}, \xi\right)>$ $n_{\oplus}-\nu$, then

$$
d\left(x_{\ominus}, \xi\right) \geq d\left(x_{\oplus}, \xi\right)-d\left(x_{\oplus}, x_{\ominus}\right)>\left(n_{\oplus}-\nu\right)-\left(n_{\oplus}-n_{\ominus}\right)=n_{\ominus}-\nu
$$

and $U_{n_{\ominus} \nu}\left(x_{\ominus}, \xi\right)=0$.

The upper bound looks alike either because of time regularization in the case $\nu=0$ and $\xi \in B\left(x_{0}, 2 \varepsilon R\right)$ or because of space regularization in the case $\xi \in \partial B\left(x_{0}, 2 \varepsilon R\right)$. In the first case, for $x_{\ominus} \in B\left(x_{0}, \varepsilon R\right)$ and $\theta_{1} R^{2} \leq n_{\ominus} \leq \theta_{2} R^{2}$

$$
\begin{aligned}
U_{n_{\ominus}}\left(x_{\ominus}, \xi\right) & \leq p_{n_{\ominus}}\left(x_{\ominus}, \xi\right) \\
& \leq \frac{C_{r} m(\xi)}{V\left(x_{\ominus}, \sqrt{n_{\ominus}}\right)} \\
& \leq \frac{C_{r} m(\xi)}{V\left(x_{\ominus}, \theta_{1} R\right)} \\
& \leq \frac{C m(\xi)}{V\left(x_{0}, 2 \varepsilon R\right)}
\end{aligned}
$$

where $C=C_{r} C_{1}^{N}$, we must apply the volume regularity $N$ times, $N$ depending on $\varepsilon$ and $\theta_{1}$. In the second case, we use the Gaussian coefficient and $d\left(x_{\ominus}, \xi\right) \geq[2 \varepsilon R]-[\varepsilon R]$,

$$
\begin{aligned}
U_{n_{\ominus}-\nu}\left(x_{\ominus}, \xi\right) & \leq p_{n_{\ominus}-\nu}\left(x_{\ominus}, \xi\right) \\
& \leq \frac{C_{r} m(\xi)}{V\left(x_{\ominus}, \sqrt{n_{\ominus}-\nu}\right)} e^{-c_{r} d\left(x_{\ominus}, \xi\right)^{2} /\left(n_{\ominus}-\nu\right)} \\
& \leq \frac{C m(\xi)}{V\left(x_{0}, 2 \varepsilon R\right)} .
\end{aligned}
$$

\subsection{Poincaré inequality.}

Theorem 3.11. Assume $H\left(C_{H}\right)$, then there exist $C_{1}, C_{2}$ and $\alpha>0$ such that $D V\left(C_{1}\right), P\left(C_{2}\right)$ and $\Delta(\alpha)$ are true.

Proof. In the comments after Theorem 1.7, we already mentioned that $H\left(C_{H}\right)$ implies a property $\Delta(\alpha)$. Then $D V\left(C_{1}\right)$ is proven as in Section 3.1. The discrete version raises new difficulties only for small 
radii but then $\Delta(\alpha)$ is sufficient. Thus we also obtain, as in Proposition 3.1 ,

$$
d(x, y)^{2} \leq n \text { implies } p_{n}(x, y) \geq \frac{c m(y)}{V(x, \sqrt{t})} .
$$

The fact that parabolic Harnack inequality implies Poincaré inequality is proven on manifolds in [30] with ideas of [18]. Take $f$ defined on $B\left(x_{0}, 2 r\right)$ and consider the Neumann problem on $B\left(x_{0}, 2 r\right)$. It may be defined this way: consider the graph $B\left(x_{0}, 2 r\right)$ with the restriction $\left.\mu\right|_{B\left(x_{0}, 2 r\right) \times B\left(x_{0}, 2 r\right)}$, it gives a kernel $p^{\prime}(x, y)$. The crucial point is that $p^{\prime}(x, y)$ has increased (comparing to $\left.p(x, y)\right)$ for $x \in \partial B\left(x_{0}, 2 r\right)$. Set $P$ the Markov operator

$$
P g(x)=\sum_{y} p^{\prime}(x, y) g(y)
$$

and denote the iteration $Q=P^{\left[4 r^{2}\right]}$. For any positive $g, P^{n} g(x)$ is a positive solution on $B\left(x_{0}, 2 r\right)$ of the parabolic equation (of $\Gamma$ ). Thus, for $x \in B\left(x_{0}, r\right)$,

$$
\begin{aligned}
\left(Q(f-(Q f)(x))^{2}\right)(x) & \geq \sum_{y \in B\left(x_{0}, r\right)} \frac{c m(y)}{V(x, 2 r)}(f(y)-(Q f)(x))^{2} \\
& \geq \frac{c}{V\left(x_{0}, 3 r\right)} \sum_{y \in B\left(x_{0}, r\right)} m(y)\left(f(y)-f_{B\left(x_{0}, r\right)}\right)^{2}
\end{aligned}
$$

because $\sum_{y \in B\left(x_{0}, r\right)} m(y)(f(y)-\lambda)^{2}$ is minimal for $\lambda=f_{B\left(x_{0}, r\right)}$. This yields

$$
\begin{aligned}
\sum_{y \in B\left(x_{0}, r\right)} m(y)\left|f(y)-f_{B\left(x_{0}, r\right)}\right|^{2} & \leq C \sum_{x \in B\left(x_{0}, 2 r\right)}\left(Q(f-(Q f)(x))^{2}\right)(x) \\
& =C\left(\|f\|_{2}^{2}-\|Q f\|_{2}^{2}\right) \\
(3.20) & \leq C\left(4 r^{2}\|\nabla f\|_{2}^{2}\right),
\end{aligned}
$$

where

$$
\|f\|_{2}^{2}=\sum_{x \in B\left(x_{0}, 2 r\right)} m^{\prime}(x) f(x)^{2}
$$

and

$$
\|\nabla f\|_{2}^{2}=\sum_{x, y \in B\left(x_{0}, 2 r\right)} \mu_{x y}|f(x)-f(y)|^{2} .
$$


The line (3.20) is a variance formula and the line (3.21) is justified by the two properties

$$
\|P f\|_{2}^{2} \leq\|f\|_{2}^{2} \quad \text { and } \quad\|f\|_{2}^{2}-\|P f\|_{2}^{2} \leq\|\nabla f\|_{2}^{2} .
$$

We give the proof of the second one which is not so widely known as the first. Note that $a^{2}-b^{2} \leq 2 a(a-b)$,

$$
\begin{aligned}
\sum_{x}\left(m^{\prime}(x)\left(f(x)^{2}-\left(\sum_{y} p^{\prime}(x, y) f(y)\right)^{2}\right)\right) \\
\quad \leq \sum_{x}\left(2 m^{\prime}(x) f(x)\left(f(x)-\left(\sum_{y} p^{\prime}(x, y) f(y)\right)\right)\right) \\
=2 \sum_{x, y}(\underbrace{m^{\prime}(x) p^{\prime}(x, y)}_{\mu_{x y}} f(x)(f(x)-f(y))) \\
=\sum_{x, y}\left(\mu_{x y}(f(x)-f(y))(f(x)-f(y))\right) .
\end{aligned}
$$

This ends the proof of Theorem 1.7.

\section{Some consequences of Harnack inequality and Gaussian estimates.}

\subsection{Hölder regularity.}

Among the immediate consequences of Harnack inequality are Liouville theorem stated in [9] because only the elliptic version is needed and Hölder regularity of solutions of the discrete parabolic equation.

Proposition 4.1. Assume $(\Gamma, \mu)$ satisfies the properties of Theorem 1.7. Then there exists $h>0$ and $C$ such that for all $x_{0} \in \Gamma, n_{0} \in \mathbb{Z}$ and $R \in \mathbb{N}$, if $u$ is a solution on $Q=\left(\mathbb{Z} \cap\left[n_{0}-2 R^{2}, n_{0}\right]\right) \times B\left(x_{0}, 2 R\right)$, $x_{1}, x_{2} \in B\left(x_{0}, R\right)$ and $n_{1}, n_{2} \in \mathbb{Z} \cap\left[n_{0}-R^{2}, n_{0}\right]$, then

$$
\left|u\left(n_{2}, x_{2}\right)-u\left(n_{1}, x_{1}\right)\right| \leq C\left(\frac{\sup \left\{\sqrt{\left|n_{2}-n_{1}\right|}, d\left(x_{1}, x_{2}\right)\right\}}{R}\right)^{h} \sup _{Q}|u| .
$$


Proof. Fix $n_{2} \geq n_{1}$ and set $Q(i)=\left(\mathbb{Z} \cap\left[n_{2}-2^{2 i}, n_{0}\right]\right) \times B\left(x_{2}, 2^{i}\right)$, $M(i)=\sup _{Q(i)} u, m(i)=\inf _{Q(i)} u$ and $\omega(i)=M(i)-m(i), 2^{i_{1}-1} \leq$ $\sup \left\{\sqrt{\left|n_{2}-n_{1}\right|}, d\left(x_{1}, x_{2}\right)\right\}<2^{i_{1}}$ and $2^{i_{2}} \leq R<2^{i_{2}+1}$. This way, $\omega\left(i_{1}\right) \geq\left|u\left(n_{2}, x_{2}\right)-u\left(n_{1}, x_{1}\right)\right|$ and $\omega\left(i_{2}\right) \leq 2 \sup _{Q}|u|$.

Set $m_{\ominus}(i)=u\left(n_{2}-2^{2 i+1}, x_{2}\right)$ and apply Harnack inequality in $Q(i+1)$ to $u-m(i+1)$ and $M(i+1)-u$

$$
\begin{gathered}
m_{\ominus}(i)-m(i+1) \leq C_{H}(m(i)-m(i+1)), \\
M(i+1)-m_{\ominus}(i) \leq C_{H}(M(i+1)-M(i)) .
\end{gathered}
$$

This yields $\omega(i) \leq\left(1-C_{H}^{-1}\right) \omega(i+1)$. Thus,

$$
\omega\left(i_{1}\right) \leq\left(1-C_{H}^{-1}\right)^{i_{2}-i_{1}} \omega\left(i_{2}\right)
$$

and the proposition follows.

\subsection{Green function.}

With the Gaussian estimates for $p_{n}$, one easily proves estimates for the Green function.

Proposition 4.2. Assume $(\Gamma, \mu)$ satisfies the properties of Theorem 1.7. Then the Green function $G(x, y)=\sum_{n=0}^{+\infty} p_{n}(x, y)$ is finite if and only if

$$
\sum_{n=0}^{+\infty} \frac{n}{V(x, n)}<+\infty
$$

and it satisfies the estimates

$$
\begin{aligned}
C^{-1} m(y) \sum_{n=d(x, y)}^{+\infty} \frac{n}{V(x, n)} & \leq G(x, y) \\
& \leq C m(y) \sum_{n=d(x, y)}^{+\infty} \frac{n}{V(x, n)}
\end{aligned}
$$

Note that condition (4.22) is satisfied or not uniformly for $x \in \Gamma$. Indeed, for $n \geq d\left(x, x^{\prime}\right), C_{1}^{-1} V(x, n) \leq V\left(x^{\prime}, n\right) \leq C_{1} V(x, n)$. On 
manifolds, the necessity of (4.22) was proved in [37]. The sufficiency and the estimates (4.23) were studied in [19], [34], [35], [36] with assumptions on the curvature. With the work [30], L. Saloff-Coste obtained them with Poincaré inequality assumption.

Proof. We use the Gaussian estimates $G\left(c_{l}, C_{l}, C_{r}, c_{r}\right)$. They yield

$$
\begin{aligned}
C^{-1} m(y) \sum_{n=d^{2}(x, y)}^{+\infty} \frac{1}{V(x, \sqrt{n})} & \leq G(x, y) \\
& \leq C m(y) \sum_{n=d^{2}(x, y)}^{+\infty} \frac{1}{V(x, \sqrt{n})}
\end{aligned}
$$

The lower bound is a consequence of

$$
G(x, y)=\sum_{n=0}^{+\infty} p_{n}(x, y) \geq \sum_{n=d^{2}(x, y)}^{+\infty} p_{n}(x, y) \geq \sum_{n=d^{2}(x, y)}^{+\infty} \frac{c_{l} m(y)}{V(x, \sqrt{n})} e^{-C_{l}}
$$

The upper bound is obtained by dividing the sum $G(x, y)$ into two parts

$$
\sum_{n=d^{2}(x, y)}^{+\infty} p_{n}(x, y) \leq C_{r} m(y) \sum_{n=d^{2}(x, y)}^{+\infty} \frac{1}{V(x, \sqrt{n})}
$$

and

$$
\begin{aligned}
\sum_{n=0}^{d^{2}(x, y)} p_{n}(x, y) & \leq \sum_{n=d(x, y)}^{d^{2}(x, y)} \frac{C_{r} m(y)}{V(x, \sqrt{n})} e^{-c_{r} d^{2}(x, y) / n} \\
& \leq C_{r} m(y) \sum_{n=d(x, y)}^{d^{2}(x, y)} \frac{1}{V(x, 2 d(x, y))} \\
\cdot & \underbrace{C_{1}\left(\frac{2 d(x, y)}{\sqrt{n}}\right)^{\log C_{1} / \log 2} e^{-c_{r} d^{2}(x, y) / n}}_{\leq \text {constant }} \\
& \leq C m(y) \sum_{n=d^{2}(x, y)}^{\frac{1}{V(x, \sqrt{n})}} .
\end{aligned}
$$


The proposition follows from (4.24) since

$$
\sum_{n=d^{2}(x, y)}^{+\infty} \frac{1}{V(x, \sqrt{n})}=\sum_{k=d(x, y)}^{+\infty} \underbrace{\#\{n \in \mathbb{N}: k \leq \sqrt{n}<k+1\}}_{=2 k+1} \frac{1}{V(x, k)} .
$$

Acknowledgements. I wish to thank L. Saloff-Coste and T. Coulhon for encouragement and useful remarks.

\section{References.}

[1] Aronson, D. G., Bounds for the fundamental solution of a parabolic equation. Bull. Amer. Math. Soc. 73 (1967), 890-896.

[2] Bombieri, E., Theory of minimal surfaces and a counter-example to the Bernstein conjecture in high dimensions. Mimeographed Notes of Lectures held at Courant Institute. New-York University, 1970.

[3] Chung, F. R. K., Yau S. T., A Harnack inequality for homogeneous graphs and subgraphs. Comm. Anal. Geom. 2 (1994), 628-639.

[4] Coulhon, T., Saloff-Coste, L., Puissances d'un opérateur régularisant. Ann. Inst. H. Poincaré, Probabilités et Statistique 26 (1990), 419-436.

[5] Coulhon, T., Saloff-Coste, L., Minorations pour les chaînes de Markov unidimensionnelles. Probab. Theor. Relat. Fields 97 (1993), 423-431.

[6] Coulhon, T., Saloff-Coste, L., Isopérimétrie sur les groupes et les variétés. Revista Mat. Iberoamericana 9 (1993), 293-314.

[7] Davies, E. B., Heat kernels and spectral theory. Cambridge University Press, 1989.

[8] Davies, E. B., Large deviations for heat kernels on graphs. J. London Math. Soc. 47 (1993), 65-72.

[9] Delmotte, T., Inégalité de Harnack elliptique sur les graphes. Colloquium Mathematicum 72 (1997), 19-37.

[10] Delmotte, T., Estimations pour les chaînes de Markov réversibles. C. R. Acad. Sci. Paris 324 (1997), 1053-1058.

[11] Fabes, E., Stroock, D., A new proof of Moser's parabolic Harnack inequality using the old ideas of Nash. Arch. Rational Mech. Anal. 96 (1986), 327-338.

[12] Grigor'yan, A., The heat equation on noncompact Riemannian manifolds. Math. USSR Sb. 72 (1992), 47-77.

[13] Grigor'yan, A., Gaussian upper bounds for the heat kernel on arbitrary manifolds. J. Differential Geom. 45 (1997), 33-52. 
[14] Hajłasz, P., Koskela, P., Sobolev meets Poincaré. C. R. Acad. Sci. Paris 120 (1995), 1211-1215.

[15] Hebisch, W., Saloff-Coste, L., Gaussian estimates for Markov chains and random walks on groups. Ann. Probab. 21 (1993), 673-709.

[16] Holopainen, I., Soardi, P. M., A strong Liouville theorem for $p$-harmonic functions on graphs. Ann. Acad. Sci. Fennicae Math. 22 (1997), 205226 .

[17] Jerison, D., The Poincaré inequality for vector fields satisfying the Hörmander 's condition. Duke Math. J. 53 (1986), 503-523.

[18] Kusuoka, S., Stroock, D., Application of Malliavin calculus, part 3. J. Fac. Sci. Univ. Tokyo, Série IA, Math. 34 (1987), 391-442.

[19] Li, P., Yau, S. T., On the parabolic kernel of the Schrödinger operator. Acta Math. 156 (1986), 153-201.

[20] Merkov, A. B., Second-order elliptic equations on graphs. Math. USSR Sb. 55 (1986), 493-509.

[21] Moser, J., On Harnack's Theorem for elliptic differential equations. Comm. Pure Appl. Math. 14 (1961), 577-591.

[22] Moser, J., A Harnack inequality for parabolic differential equations. Comm. Pure Appl. Math. 17 (1964), 101-134. Correction in 20 (1967), 231-236.

[23] Moser, J., On a pointwise estimate for parabolic differential equations. Comm. Pure Appl. Math. 24 (1971), 727-740.

[24] Nash, J., Continuity of solutions of parabolic and elliptic equations. Amer. J. Math. 80 (1958), 931-954.

[25] Pang, M. M. H., Heat kernels of graphs. J. London Math. Soc. 47 (1993), 50-64.

[26] Rigoli, M., Salvatori, M., Vignati, M., A global Harnack inequality on graphs and some related consequences. Preprint.

[27] Russ, E., Riesz transforms on graphs for $1 \leq p \leq 2$. To appear in Math. Scandinavia.

[28] Saloff-Coste, L., Uniformly elliptic operators on Riemannian manifolds. J. Differential Geom. 36 (1992), 417-450.

[29] Saloff-Coste, L., A note on Poincaré, Sobolev and Harnack inequalities. Internat. Math. Res. Notices 2 (1992), 27-38.

[30] Saloff-Coste, L., Parabolic Harnack inequality for divergence form second order differential operators. Potential analysis 4 (1995), 429-467.

[31] Saloff-Coste, L., Lectures on finite Markov chains. Cours de l'Ecole d'Eté de Probabilités de Saint-Flour, 1996.

[32] Saloff-Coste, L., Stroock, D., Opérateurs uniformément sous-elliptiques sur les groupes de Lie. J. Funct. Anal. 98 (1991), 97-121. 
[33] Stroock, D. W., Zheng W., Markov chain approximations to symmetric diffusions. Ann. Inst. H. Poincaré 33 (1997), 619-649.

[34] Varopoulos, N., The Poisson kernel on positively curved manifolds. J. Funct. Anal. 44 (1981), 359-380.

[35] Varopoulos, N., Green's function on positively curved manifolds. $J$. Funct. Anal. 45 (1982), 109-118.

[36] Varopoulos, N., Green's function on positively curved manifolds, II. $J$. Funct. Anal. 49 (1982), 170-176.

[37] Varopoulos, N., Potential theory and diffusion on Riemaniann manifolds. Conference on harmonic analysis in honor of Antoni Zygmund, Vol. I, II, Wadsworth Math. Ser., Wadsworth, Belmont, Calif. (1983), 821-837.

[38] Varopoulos, N., Small time Gaussian estimates of the heat diffusion kernel, Part 1: the semigroup technique. Bull. Sci. Math. 113 (1989), 253-277.

[39] Varopoulos, N., Coulhon, T., Saloff-Coste, L., Analysis and geometry on groups. Cambridge University Press, 1993.

Recibido: 26 de noviembre de 1.997

Thierry Delmotte Eidgenössische Technische Hochschule Zürich

D-Math HG-649.1

ETH Zentrum

CH-8092 Zürich, SWITZERLAND

delmotte@math.ethz.ch 